\title{
A Polyakov Action on Riemann Surfaces (II)
}

\author{
Roberto Zucchini * \\ Laboratoire de Physique Théorique ENSLAPP, Chemin de Bellevue, B.P. 110, \\ F-74941 Annecy-le-Vieux, France, and Dipartimento di Fisica, Università degli Studi di Bologna, \\ V. Irnerio 46, I-40126 Bologna, Italy
}

Received December 6, 1991; in revised form September 20, 1992

\begin{abstract}
We continue the model independent study of the Polyakov action on an arbitrary compact surface without boundary of genus larger than 2 as the general solution of the relevant conformal Ward identity. A general formula for the Polyakov action and an explicit calculation of the energy-momentum tensor density is provided. It is further shown how Polyakov's SL $(2, \mathbf{C})$ symmetry emerges in a curved base surface.
\end{abstract}

\section{Introduction}

Since the mid-eighties, a large body of theoretical and mathematical literature has been devoted to the study of two-dimensional conformal field theories on Riemann surfaces without boundary $[1,2]$. These models are relevant in string theory and in the analysis of the 2-dimensional statistical system obeying certain periodic boundary conditions at criticality. In more recent times, the dependence on the background geometry has been exploited to obtain effective actions for two-dimensional quantum gravity. This has led to exciting developments in non-critical string theory $[3,4]$ and may conceivably shed some light on the quantization programme of higher dimensional gravity.

Most of the studies on the subject are concerned with Lagrangian field theories on a two-dimensional Riemannian manifold $(\Sigma, g)$ which are both Weyl and diffeomorphism invariant at the classical level [5-7]. The quantization program is carried out by means of a diffeomorphism invariant renormalization scheme, typically the $\zeta$ function scheme. In general, however, a Weyl anomaly is produced in this way. Let us see this in greater detail. Parametrize the metric $g$ as usual as

$$
g=\exp (\phi) \varrho_{0}|d z+\mu d \bar{z}|^{2}
$$

[8-10]. Here, $z, \bar{z}$ are the coordinates of a reference holomorphic coordinate covering. $\phi$ is the Weyl phase. $\mu$ is the Beltrami differential characterizing the conformal class

* Present address: Dipartimento di Fisica, Università di Bologna, Bologna, Italy 
of $g . \varrho_{0}$ is a background metric and is needed to write $g$ invariantly. The infinitesimal action of the Weyl and diffeomorphism symmetry on the above fields is given by the nilpotent Slavnov operator $s$ and is expressed in terms of the respective ghost fields $F$ and $c$ :

$$
\begin{aligned}
s \phi & =F+(c \partial+\bar{c} \bar{\partial}) \phi+\left(\nabla_{0}+\bar{\mu} \bar{\partial}\right) c+\left(\bar{\nabla}_{0}+\mu \partial\right) \bar{c} \\
s \mu & =(\bar{\partial}-\mu \partial+(\partial \mu)) C, \quad C=c+\mu \bar{c} \\
s F & =(c \partial+\bar{c} \bar{\partial}) F \\
s c & =(c \partial+\bar{c} \bar{\partial}) c
\end{aligned}
$$

where $\nabla_{0}$ is the covariant derivative associated to the Riemann-Christoffel-Ricci-Levi Civita connection $\gamma_{0}$ of $\varrho_{0}$

$$
\gamma_{0}=\partial \ln \varrho_{0}
$$

The background $\varrho_{0}$ itself is invariant:

$$
s \varrho_{0}=0 .
$$

The diffeomorphism invariant effective action $\Gamma_{D}$ is a functional of the metric $g$ and thus, by (1.1), of the fields $\phi, \mu, \bar{\mu}$, and the background $\varrho_{0}$. It obeys the Ward identity

$$
s \Gamma_{D}\left(\phi, \mu, \bar{\mu} ; \varrho_{0}\right)=k \mathcal{A}_{(}\left(F ; \phi, \mu, \bar{\mu} ; \varrho_{0}\right),
$$

where

$$
\begin{aligned}
& \mathscr{A}\left(F ; \phi, \mu, \bar{\mu} ; \varrho_{0}\right) \\
& =\frac{-1}{12 \pi} \int_{\Sigma} \frac{d \bar{z} \wedge d z}{2 i} F\left\{\partial \bar{\partial} \phi+\partial \bar{\partial} \ln \varrho_{0}\right. \\
& \left.\quad-(\partial-\bar{\mu} \bar{\partial}-(\bar{\partial} \bar{\mu})) \frac{\nabla_{0} \mu+\partial \phi \mu}{1-\mu \bar{\mu}}-(\bar{\partial}-\mu \partial-(\partial \mu)) \frac{\bar{\nabla}_{0} \bar{\mu}+\bar{\partial} \phi \bar{\mu}}{1-\mu \bar{\mu}}\right\}
\end{aligned}
$$

[5-7]. Here, a Weyl anomaly appears whose strength is measured by a real coefficient $k$. The form of such anomaly is universal. With a conventional normalization, $k$ coincides with the central charge of the model under consideration. The Weyl anomaly can be eliminated by either i) constraining the field content of the model so that the total central charge vanishes, as in the case of string theory [5], or ii) subtracting from the effective action a suitable local counterterm that absorbs the Weyl anomaly at the cost of creating a diffeomorphism anomaly [11]. The form of such counterterm is also universal. Up to a factor $k$, the counterterm is given by

$$
\Delta \Gamma\left(\phi, \mu, \bar{\mu} ; \varrho_{0}, \mathscr{B}_{0}, \overline{\mathscr{B}}_{0}\right)=\Delta_{1} \Gamma\left(\phi, \mu, \bar{\mu} ; \varrho_{0}\right)+\Delta_{2} \Gamma\left(\phi, \mu, \bar{\mu} ; \varrho_{0}, \mathscr{R}_{0}, \overline{\mathscr{B}}_{0}\right),
$$

where

$$
\begin{aligned}
& \Delta_{1} \Gamma\left(\phi, \mu, \bar{\mu} ; \varrho_{0}\right)=\frac{-1}{12 \pi} \int_{\Sigma} \frac{d \bar{z} \wedge d z}{2 i}\left\{\frac{1}{2(1-\mu \bar{\mu})}(\partial-\bar{\mu} \bar{\partial}) \phi(\bar{\partial}-\mu \partial) \phi\right. \\
& \left.-\phi\left[\partial \bar{\partial} \ln \varrho_{0}-(\partial-\bar{\mu} \bar{\partial}-(\bar{\partial} \bar{\mu})) \frac{\nabla_{0} \mu}{1-\mu \bar{\mu}}-(\bar{\partial}-\mu \partial-(\partial \mu)) \frac{\bar{\nabla}_{0} \bar{\mu}}{1-\mu \bar{\mu}}\right]\right\} \\
& \Delta_{2} \Gamma\left(\phi, \mu, \bar{\mu} ; \varrho_{0}, \mathscr{R}_{0}, \overline{\mathscr{R}}_{0}\right)=\frac{1}{12 \pi} \int_{\Sigma} \frac{d \bar{z} \wedge d z}{2 i}\left\{\mu\left(\mathscr{R}_{0}-r_{0}\right)+\bar{\mu}\left(\overline{\mathscr{R}}_{0}-\bar{r}_{0}\right)\right. \\
& \left.-\frac{1}{1-\mu \bar{\mu}}\left[\nabla_{0} \mu \bar{\nabla}_{0} \bar{\mu}-\frac{1}{2} \bar{\mu}\left(\nabla_{0} \mu\right)^{2}-\frac{1}{2} \mu\left(\bar{\nabla}_{0} \bar{\mu}\right)^{2}\right]\right\}
\end{aligned}
$$


and $r_{0}$ is the projective connection

$$
r_{0}=\partial \gamma_{0}-\frac{1}{2} \gamma_{0}^{2}
$$

$\mathscr{B}_{0}$ is a holomorphic projective connection in the conformal structure of the reference coordinate covering $z, \bar{z}$ and satisfies

$$
s \mathscr{B}_{0}=0 \text {. }
$$

$\mathscr{R}_{0}$ ensures the correct conformal covariance of the integrand in the right hand side of Eq. (1.10c). The Weyl invariant effective action $\Gamma_{W}$ is obtained by adding the counterterm to $\Gamma_{D}[11]$ :

$$
\Gamma_{W}\left(\mu, \bar{\mu} ; \mathscr{R}_{0}, \overline{\mathscr{R}}_{0}\right)=\Gamma_{D}\left(\phi, \mu, \bar{\mu} ; \varrho_{0}\right)+k \Delta \Gamma\left(\phi, \mu, \bar{\mu} ; \varrho_{0}, \mathscr{R}_{0}, \overline{\mathscr{R}}_{0}\right) .
$$

$\Gamma_{W}$ depends on the fields $\mu, \bar{\mu}$ and the background $\mathscr{B}_{0}$ and $\overline{\mathscr{B}}_{0}$. Crucially, Weyl invariance ensures that no dependence on $\varrho_{0}$ occurs. $\Gamma_{W}$ obeys the Ward identity

$$
s \Gamma_{W}\left(\mu, \bar{\mu} ; \mathscr{R}_{0}, \overline{\mathscr{B}}_{0}\right)=k \cdot \mathscr{A}\left(C ; \mu ; \mathscr{B}_{0}\right)+k \overline{\mathscr{A}\left(C ; \mu ; \mathscr{B}_{0}\right)},
$$

where

$$
\mathscr{b}\left(C ; \mu ; \mathscr{B}_{0}\right)=\frac{1}{12 \pi} \int_{\Sigma} \frac{d \bar{z} \wedge d z}{2 i} C\left(\partial^{3}+2 R_{0} \partial+\left(\partial \mathscr{R}_{0}\right)\right) \mu
$$

$[12,13]$. The Weyl anomaly has been traded for a diffeomorphism anomaly whose strength is again measured by the central charge $k$. The salient feature of the diffeomorphism anomaly is that it is chirally split: it is the sum of two contributions each of which depends on only one of the pairs $(C, \mu)$ and $(\bar{C}, \bar{\mu})$. This fact is intimately related to the holomorphic factorization property of $\Gamma_{W}$ which reads

$$
\Gamma_{W}\left(\mu, \bar{\mu} ; \mathscr{R}_{0}, \overline{\mathscr{B}}_{0}\right)=\Gamma_{P}\left(\mu ; \mathscr{B}_{0}\right)+\overline{\Gamma_{P}\left(\mu ; \mathscr{B}_{0}\right)},
$$

$[14,15]$. The functional $\Gamma_{P}$ is called the Polyakov action of the model under consideration [16]. This is a most fundamental object. It depends holomorphically on the Beltrami differential $\mu$ and the background $\mathscr{B}_{0}$ and satisfies the chiral conformal Ward identity

$$
s \Gamma_{P}\left(\mu ; \mathscr{B}_{0}\right)=k \cdot \mathscr{b}\left(C ; \mu ; \mathscr{B}_{0}\right) .
$$

Combinations of Polyakov actions can be used to construct explicitly chiral conformal field theories. Last, but not least, any Polyakov action may serve as a "classical" action for $2 D$ quantum gravity in the so-called light cone gauge [16-20]. Such action being non-local, the quantization programme is highly non-trivial.

The independence of $\Gamma_{P}\left(\mu ; \mathscr{R}_{0}\right)$ from $\phi$ and $\varrho_{0}$ entails that such functional depends only on the background conformal geometry parametrized by the Beltrami differential $\mu$. This suggests that a natural scheme for the study of 2-dimensional conformal field theory on Riemann surfaces should rely ab initio and exclusively on conformal geometry $[21,22]$. It would also be preferable if such a scheme were non-Lagrangian in nature, in order to allow for a wider range of applications and not bias our understanding of the subject. For such reasons, I shall adopt a somewhat axiomatic point of view and postulate a few general properties of $\Gamma_{P}\left[\mu, \mathscr{B}_{0}\right]$ which have been shown in a number of examples but are expected to hold in general. Then, I shall try to prove general theorems by relying exclusively on those properties. In practice, this amounts to find the most general solution of the Ward identity (1.16) that 
depends holomorphically on $\mu$. The advantage of this approach is that all kinematical implications of the conformal symmetry are explicitly and fully worked out and neatly separated from model dependent features.

It can be shown in the framework of renormalized field theory based on locality and power counting that, in the infinite plane, the conformal Ward identity (1.16) has a unique solution [23, 24]. This has been found by Polyakov in ref. [16, Eq. (5)]. The generalization of Polyakov's formula to a compact Riemann surface without boundary has been worked out in ref. [25] (see also [26]). In this case, however, the solution is non-unique since it is defined up to addition of an arbitrary local holomorphic function on a non-trivial Teichmueller space. In this paper, I shall continue the study of the Polyakov action on a compact Riemann surface without boundary begun in ref. [25]. Here, I have found an alternative but equivalent expression of the Polyakov action which is more suitable for the study of its formal properties. I have also reached a better understanding of the geometrical structure lying beneath the whole construction and of the mechanism responsible for the appearance of the $\operatorname{SL}(2, \mathrm{C})$ symmetry in a curved base surface.

The plan of this paper is as follows. In Sect. 2, I shall review the basic definitions and results of conformal and projective geometry which will be used throughout. These notions are already known to the scholars working in the field and have been recalled for the purpose of setting the notation and for later reference. In Sect. 3, the properties of the infinitesimal action of the diffeomorphism group on the relevant fields are briefly expounded and the Slavnov operator is introduced. In Sect. 4, the general formula of the Polyakov action is given and shown to solve the conformal Ward identity (1.16) and the calculation of the energy-momentum tensor is illustrated. Finally, in Sect. 5, I shall review the basic formal properties of the Polyakov action, show how the $\operatorname{SL}(2, \mathrm{C})$ symmetry emerges in this context and review the problems that are still open.

\section{Higher Genus Surfaces and Their Conformal and Projective Geometry}

Let $\Sigma$ be a connected compact differentiable surface without boundary of genus $g \geq 2$. Let

$$
\pi: \Sigma_{\pi} \rightarrow \Sigma
$$

be a universal covering of $\Sigma$ and Deck $_{\pi}$ its universal covering group. As is wellknown, $\Sigma_{\pi}$ is a connected simply connected differentiable surface and $\pi$ is a local diffeomorphism. For this reason, it is harmless and convenient not to distinguish notationally between any sheaf theoretic structure on $\Sigma$ (e.g. a coordinate structure, a line bundle, a section of a line bundle, etc.) and its pull-back to $\Sigma_{\pi}$ by $\pi$.

Let $\mathbf{a}$ be a reference conformal structure, i.e. a maximal atlas of local coordinates with holomorphic coordinate changes. I shall denote a generic coordinate of a by $z$. All relations of this paper will be written in terms of the coordinates of $\mathbf{a}$, unless otherwise stated.

Let $k$ be the a-holomorphic cotangent line bundle of $\Sigma$ and $l$ an a-holomorphic line bundle on $\Sigma$ such that $l^{\otimes 2}=k$. Let $O$ be a non-empty open subset of $\Sigma$ and $p, q$ half-integer numbers. A conformal field $\psi$ of weights $p, q$ on $O$ is a smooth section of $l^{\otimes 2 p} \otimes i^{\otimes 2 q}$ on $\pi^{-1}(O)$. The infinite dimensional complex vector space of all such fields will be denoted by $C_{\pi}^{p, q}(O)$. A (scalar) multiplier $\chi$ on $O$ is a map that 
associates to any element $\gamma$ of $\operatorname{Deck}_{\pi}$ a nowhere vanishing element $\chi(\gamma)$ of $C_{\pi}^{0,0}(O)$ such that

$$
\begin{gathered}
\chi\left(\gamma_{1} \circ \gamma_{2}\right)=\chi\left(\gamma_{1}\right) \circ \gamma_{2} \cdot \chi\left(\gamma_{2}\right), \\
\chi\left(\gamma_{1}^{-1} \circ \gamma_{2}^{-1} \circ \gamma_{1} \circ \gamma_{2}\right)=1
\end{gathered}
$$

for any two $\gamma_{1}, \gamma_{2}$ in $\operatorname{Deck}_{\pi}$. The map $\gamma \rightarrow \varepsilon(\gamma)=1$ defines a multiplier called the trivial multiplier. The multipliers clearly form a group $G_{\pi}(O)$ under pointwise multiplication. A conformal field $\psi$ of $C_{\pi}^{p, q}(O)$ has definite monodromy if there is a multiplier $\chi$ such that

$$
\gamma^{*} \psi=\chi(\gamma) \cdot \psi
$$

for any $\gamma$ in $\operatorname{Deck}_{\pi}$, where $\gamma^{*} \psi$ denotes the pull-back action of $\gamma$ on $\psi$. The polydromic fields with multiplier $\chi$ form an infinite dimensional complex vector space $C^{p, q}(O, \chi)$. The monodromic conformal fields are those polydromic fields whose multiplier is the trivial multiplier. They form an infinite dimensional complex vector space $C^{p, q}(O)$.

A Beltrami differential $\mu$ on $\Sigma$ is an element of $C^{-1,1}(\Sigma)$ satisfying the boundedness condition $\sup _{\Sigma}|\mu|<1$. The set $\operatorname{Beltr}(\Sigma)$ of all Beltrami differentials on $\Sigma$ has an obvious structure of topologically contractible complex Banach manifold.

$\operatorname{Beltr}(\Sigma)$ parametrizes the set of all conformal structures on $\Sigma$. Indeed, to any Beltrami differential $\mu$ in $\operatorname{Beltr}(\Sigma)$, there is associated a conformal structure $\mathbf{A}(\mu)$ on $\Sigma$ whose generic coordinate $Z$ is a local solution of the Beltrami equation

$$
(\bar{\partial}-\mu \partial) Z=0
$$

obeying the local invertibility condition

$$
\partial Z \bar{\partial} \bar{Z}-\partial \bar{Z} \bar{\partial} Z>0 \text {. }
$$

Conversely, $\mathbf{A}(\mu)$ determines $\mu$ through the local relation

$$
\mu=\bar{\partial} Z / \partial Z
$$

where $Z$ is a generic coordinate $\mathbf{A}(\mu)$. In such parametrization, the following useful identities hold:

$$
\begin{aligned}
& d Z=\partial Z(d z+\mu d \bar{z}) \\
& \partial_{\bar{Z}}=\frac{1}{\bar{\partial} \bar{Z}(1-\mu \bar{\mu})}(\bar{\partial}-\mu \partial) .
\end{aligned}
$$

In particular, $\mathbf{A}(0)=\mathbf{a}$ : the reference conformal structure corresponds to the vanishing Beltrami differential. For the proof of these statements and relations, see ref. [8].

A projective connection $\mathscr{B}$ on $\Sigma$ is an assignment to any coordinate $z$ of a of a smooth function $\mathscr{B}$ defined in the domain of $z$ with the following properties. On the overlapping domains of $z$ and $z^{\prime}$, one has

$$
\mathscr{R}^{\prime}=\left(\partial^{\prime} z\right)^{2}\left[\mathscr{R}-\left\{z^{\prime}, z\right\}\right],
$$

where $\{f, \zeta\}$ denotes the Schwarzian derivative

$$
\{f, \zeta\}=\partial_{\zeta}^{2} \ln \partial_{\zeta} f-\frac{1}{2}\left(\partial_{\zeta} \ln \partial_{\zeta} f\right)^{2}=-2\left(\partial_{\zeta} f\right)^{\frac{1}{2}} \partial_{\zeta}^{2}\left(\partial_{\zeta} f\right)^{-\frac{1}{2}}
$$


Further, there exists a Beltrami differential $\mu$ in $\operatorname{Beltr}(\Sigma)$ such that $\mathscr{R}$ is $\mu$ holomorphic, that is

$$
(\bar{\partial}-\mu \partial-2(\partial \mu)) \mathscr{B}=\partial^{3} \mu .
$$

I shall denote by $\operatorname{Proj}(\Sigma, \mu)$ the set of all projective connections $\mathscr{R}$ of such type. A given projective connection $\mathscr{R}$ can be subordinated to several Beltrami differentials, for the operator $\partial^{3}+2 \mathscr{B} \partial+(\partial \mathscr{B})$ appearing in (2.12) has zero modes. For such a reason, it is convenient to introduce a fiber bundle $\operatorname{Schw}(\Sigma)$ with base $\operatorname{Beltr}(\Sigma)$ and fiber $\operatorname{Proj}(\Sigma, \mu)$ at a point $\mu$ of $\operatorname{Beltr}(\Sigma)$. This fiber bundle has a natural structure of complex Banach manifold. For further details, see $[8,27]$.

To any element $\mathscr{B}$ of $\operatorname{Proj}(\Sigma, \mu)$, there is canonically associated a projective structure $\mathbf{P}(\mu, \mathscr{R})$ subordinated to $\mathbf{A}(\mu)$. By definition, this is a maximal coordinate covering contained in $\mathbf{A}(\mu)$ whose transition functions are restrictions of elements of the Moebius group PSL(2,C). A generic coordinate $Z$ of $\mathbf{P}(\mu, \mathscr{B})$ satisfies, besides (2.5)-(2.6), the equation

$$
\left(\partial^{2}+(1 / 2) \mathscr{R}\right)(\partial Z)^{-\frac{1}{2}}=0
$$

[27]. Conversely, $\mathbf{P}(\mu, \mathscr{R})$ determines $\mu$ and $\mathscr{R}$ through (2.7) and the relation

$$
\mathscr{R}=\{Z, z\},
$$

where $Z$ is a generic coordinate of $\mathbf{P}(\mu, \mathscr{R})$. Thus, $\operatorname{Schw}(\Sigma)$ parametrizes the set of all projective structures on $\Sigma$ [27].

Let $\mu$ be an element of $\operatorname{Beltr}(\Sigma), O$ an open subset of $\Sigma$ and $j$ a half-integer number. A $\mu$-holomorphic $j$-differential $\phi$ on $O$ is an element of $C_{\pi}^{j, 0}(O)$ obeying the Beltrami type equation

$$
(\bar{\partial}-\mu \partial-j(\partial \mu)) \phi=0 \quad \text { on } \quad O .
$$

Let $H_{\pi}^{\jmath}(O, \mu)$ be the infinite dimensional complex vector space of all such differentials. A $\mu$-holomorphic multiplier $\chi$ on $O$ is an element of $G_{\pi}(O)$ such that

$$
(\bar{\partial}-\mu \partial) \chi(\gamma)=0 \quad \text { on } \quad O
$$

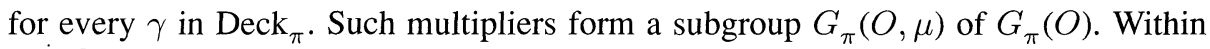
$H_{\pi}^{j}(O, \mu)$, the differentials with defined monodromy are particularly relevant. The multipliers $\chi$ pertaining $\mu$-holomorphic polydromic differentials must, for consistency, be themselves $\mu$-holomorphic. The $\mu$-holomorphic polydromic differentials on $O$ with $\mu$-holomorphic multiplier $\chi$ form a generally infinite dimensional complex vector space $H^{j}(O, \chi, \mu)$. The monodromic differentials form a generally infinite dimensional complex vector space $H^{j}(O, \mu)$. Any $\mu$-holomorphic $j$-differential $\phi$ on $O$ represents a holomorphic $j$-differential ${ }^{\theta} \phi$ on $O$ in the conformal structure $\mathbf{A}(\mu)$ and viceversa. Locally, ${ }^{\theta} \phi=(\partial Z)^{-j} \phi$ for a given coordinate $Z$ of $\mathbf{A}(\mu)$ [8].

Given an open subset (ब) $\operatorname{Beltr}(\Sigma)$ it is possible to choose for any $\mu$ in $(2$ a coordinate $Z(\mu)$ of $\mathbf{A}(\mu)$ depending holomorphically on $\mu$, i.e. $Z(\mu)$ is a continuous function of $\mu$ in $\odot$ such that

$$
\frac{\delta Z(\mu)}{\delta \bar{\mu}}=0
$$

Such coordinates are often employed. Note that in general the domain of $Z(\mu)$ depends on $\mu$. 
One may also consider maps associating to any $\mu$ from an open subset $\mathscr{O}$ of $\operatorname{Beltr}(\Sigma)$ an element $\mathscr{B}(\mu)$ of $\operatorname{Proj}(\Sigma, \mu)$. If a map $\mathscr{R}$ of such type is continuous and

$$
\frac{\delta \mathscr{R}(\mu)}{\delta \bar{\mu}}=0,
$$

then $\mathscr{R}$ defines a local holomorphic section $\mathscr{R}$ of $\operatorname{Schw}(\Sigma)$ on $\mathscr{P}$.

If the Beltrami differential $\mu$ is varied, a differential $\phi$ of $H_{\pi}^{j}(O(\mu), \mu)$ may be thought of as a functional of $\mu$. To emphasize this dependence, I shall write $\phi(\mu)$. In this paper, I shall consider only differentials depending holomorphically on $\mu$, i.e. depending continuously on $\mu$ and such that

$$
\frac{\delta \phi(\mu)}{\delta \bar{\mu}}=0 .
$$

Similarly, if $\mu$ is varied, a multiplier $\chi$ of $G_{\pi}(O(\mu), \mu)$ can be thought as a functional of $\mu$. As for holomorphic differentials, I shall consider only multipliers $\chi(\mu)$ depending holomorphically on $\mu$, i.e. depending continuously on $\mu$ and such that

$$
\frac{\delta \chi(\mu)(\gamma)}{\delta \bar{\mu}}=0
$$

for any $\gamma$ in $\operatorname{Deck}_{\pi}$. If $\phi(\mu)$ has defined monodromy with multiplier $\chi(\mu)$ and (2.19) holds, then, for consistency, $\chi(\mu)$ must satisfy (2.20).

One can define several vector bundles on $\operatorname{Beltr}(\Sigma)$ whose fiber at a point $\mu$ in $\operatorname{Beltr}(\Sigma)$ is a vector space of $\mu$-holomorphic $j$-differentials on $\Sigma$. In general, these vector bundles carry a natural complex structure. A local holomorphic section $\phi$ of one of such bundles is defined as an assignment to any $\mu$ from an open subset (?) of $\operatorname{Beltr}(\Sigma)$ of a differential $\phi(\mu)$ of $H_{\pi}^{j}(\Sigma, \mu)$ satisfying (2.19). For the following treatment, it turns out to be useful to introduce a group bundle $\mathscr{G}_{\pi}$ over $\operatorname{Beltr}(\Sigma)$ whose fiber at a point $\mu$ in $\operatorname{Beltr}(\Sigma)$ is $G_{\pi}(\Sigma, \mu) . \mathscr{G}_{\pi}$ is called the multiplier bundle. $\mathscr{G}_{\pi}$ has a natural complex structure. A local holomorphic section $\chi$ of $\mathscr{G}_{\pi}$ is an assignment to any $\mu$ from an open subset $\sigma$ of $\operatorname{Beltr}(\Sigma)$ of a multiplier $\chi(\mu)$ of $G_{\pi}(\Sigma, \mu)$ satisfying (2.20). Given a holomorphic section $\chi$ of $\mathscr{G}_{\pi}$, let $\mathscr{H}^{3}(\Sigma, \chi)$ be the holomorphic vector bundle over $\operatorname{Beltr}(\Sigma)$ whose fiber at $\mu$ is the space $H^{j}(\Sigma, \chi(\mu), \mu)$. It is assumed that the dimension of $H^{\jmath}(\Sigma, \chi(\mu), \mu)$ is independent from the Beltrami differential $\mu$. When $\chi$ is the trivial multiplier $\varepsilon$, I shall use the shorthand $\mathscr{H}^{j}(\Sigma)$ to denote the vector bundle defined above.

\section{The Diffeomorphism Group and its Infinitesimal Action}

Let $\operatorname{Diff}_{c}(\Sigma)$ denote the group of homotopically trivial orientation preserving diffeomorphisms of $\Sigma$. A given element $f$ of $\operatorname{Diff}_{c}(\Sigma)$ admits denumerably many lifts to $\Sigma_{\pi}$ by $\pi$. However, there is a unique lift of $f$, which I shall denote by the same symbol, such that

$$
f \circ \gamma=\gamma \circ f
$$

for any $\gamma$ in $\operatorname{Deck}_{\pi}$ [27]. This choice of lifting will be assumed throughout when considering the pullback action of the diffeomorphism group $\operatorname{Diff}_{c}(\Sigma)$ on polydromic conformal fields.

The diffeomorphism group $\operatorname{Diff}_{c}(\Sigma)$ acts on $\operatorname{Beltr}(\Sigma), \operatorname{Schw}(\Sigma)$ and the various vector bundles on $\operatorname{Beltr}(\Sigma)$ defined in the former section. The action of the diffeomorphism group on a coordinate structure is defined standardly: for any element $f$ of 
$\operatorname{Diff}_{c}(\Sigma)$ each coordinate $Z$ of the structure is mapped into $Z \circ f$. Through (2.7) and (2.14), this induces a free right holomorphic action on $\operatorname{Beltr}(\Sigma)$ and $\operatorname{Schw}(\Sigma)$. The holomorphic action of $\operatorname{Diff}_{c}(\Sigma)$ on $\operatorname{Beltr}(\Sigma)$ can be extended to all holomorphic fiber bundles on it by making $\operatorname{Diff}_{c}(\Sigma)$ act on the fibers by pull-back, the resulting action being similarly right, free and holomorphic. Among the holomorphic sections of these bundles, those which are invariant under the action of $\operatorname{Diff}_{c}(\Sigma)$ are particularly relevant. For further details, see refs. [8, 27].

In the following treatment, one is interested in the infinitesimal action of the diffeomorphism group Diff ${ }_{c}(\Sigma)$ on the relevant field functionals. Let $\mathbf{E}$ be the space of all such functionals. $\operatorname{Diff}_{c}(\Sigma)$ acts on $\mathbf{E}$ by a right action (e.g. pull back). To express the infinitesimal action of $\operatorname{Diff}_{c}(\Sigma)$ on $\mathbf{E}$, one introduces the space $\bigwedge^{*}$ Lie Diff $c(\Sigma)^{\vee} \otimes \mathbf{E}$, the tensor product of the exterior algebra of the dual of the Lie algebra of $\operatorname{Diff}_{c}(\Sigma)$ with $\mathbf{E}$. $\mathbf{E}$ itself may be identified with $\bigwedge^{0} \operatorname{Lie}^{\operatorname{Diff}_{c}}(\Sigma)^{\vee} \otimes \mathbf{E}$. The infinitesimal action of $\operatorname{Diff}_{c}(\Sigma)$ on $\mathbf{E}$ is given by the Slavnov operator $s$, which

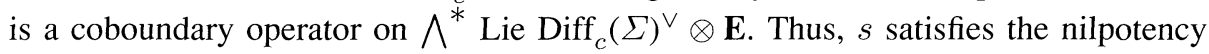
relation

$$
s^{2}=0 \text {. }
$$

The exterior algebra $\bigwedge^{*}$ Lie $\operatorname{Diff}_{c}(\Sigma)^{\vee}$ is generated by the diffeomorphism ghost field $c$ and its complex conjugate $\bar{c}:\langle c(P), \xi\rangle=\xi(P)$ for $\xi$ in $C^{-1,0}(\Sigma)$ and $P$ in $\Sigma$ and similarly for $\bar{c}$. $c$ belongs to $\bigwedge^{1}$ Lie Diff $_{c}(\Sigma)^{\vee}$ and behaves as an anticommuting conformal field of weight $-1,0$. It further obeys the structure equation

$$
s c=(c \partial+\bar{c} \bar{\partial}) c .
$$

Similar statements hold for $\bar{c}$. See [8] for further details.

The action of $s$ can be easily computed by linearizing the finite right action of $\operatorname{Diff}_{c}(\Sigma)$ on fields at the identity. For a given Beltrami differential $\mu$ in $\operatorname{Beltr}(\Sigma)$, the relevant combination of ghost fields is

$$
C(\mu)=c+\mu \bar{c} .
$$

$C(\mu)$ exhibits a distinguished complex structure of Lie $\operatorname{Diff}_{c}(\Sigma)$ [8]. The action of $s$ on $\mu$ is given by

$$
s \mu=(\bar{\partial}-\mu \partial+(\partial \mu)) C(\mu) .
$$

By combining (3.3) and (3.5), one finds further that $C(\mu)$ obeys the structure equation

$$
s C(\mu)=C(\mu) \partial C(\mu) .
$$

Equation (3.5) is one of the most important relations in the following analysis. By using (3.5) and (3.6), it is straightforward to check that the nilpotency condition (3.2) is fulfilled. See ref. [8] for further details.

As indicated above, the coordinates $Z(\mu)$ of the conformal structure $\mathbf{A}(\mu)$ transform by pull-back under $\operatorname{Diff}_{c}(\Sigma)$. This fact is equivalent to the relation

$$
s Z(\mu)=C(\mu) \partial Z(\mu) \text {. }
$$

For any $\operatorname{Diff}_{c}(\Sigma)$-invariant holomorphic section $\mathscr{R}$ of $\operatorname{Schw}(\Sigma)$, one has

$$
s \mathscr{R}(\mu)=\left(\partial^{3}+2 \mathscr{R}(\mu) \partial+(\partial \mathscr{R}(\mu))\right) C(\mu) .
$$

Conversely, if a holomorphic section $\mathscr{B}$ of $\operatorname{Schw}(\Sigma)$ satisfies (3.8), then $\mathscr{B}$ is $\operatorname{Diff}_{c}(\Sigma)$-invariant. 
Next, let $\phi$ be a local $\operatorname{Diff}_{c}(\Sigma)$-invariant holomorphic section of a vector bundle on $\operatorname{Beltr}(\Sigma)$ of holomorphic $j$-differentials. Then, the action of $s$ on $\phi$ is given by

$$
s \phi(\mu)=C(\mu) \partial \phi(\mu)+j \partial C(\mu) \phi(\mu) .
$$

This action has the property that $\langle s \phi(\mu), \xi\rangle=\mathscr{L}_{\xi} \phi(\mu)$, where $\mathscr{L}_{\xi}$ denotes the Lie derivative with respect the vector field $\xi$ of $C^{-1,0}(\Sigma)$. The fact that $s \phi(\mu)$ is expressed in terms of $C(\mu)$ depends crucially on the $\mu$-holomorphy condition (2.15). Further, if a holomorphic section $\phi$ is such that (3.9) holds, then $\phi$ is Diff ${ }_{c}(\Sigma)$-invariant. If $\chi$ a $\operatorname{Diff}_{c}(\Sigma)$-invariant holomorphic section of the multiplier bundle $\mathscr{G}_{\pi}$, then

$$
s \chi(\mu)=C(\mu) \partial \chi(\mu) .
$$

Conversely, if (3.10) holds, the $\chi$ is $\operatorname{Diff}_{c}(\Sigma)$-invariant.

\section{The Polyakov Action on a Riemann Surface: Explicit Expression and Properties}

As explained in the introduction, a Polyakov action on $\Sigma$ is any continuous functional $\Gamma(\mu)$ defined for $\mu$ varying in some open subset $(2$ of $\operatorname{Beltr}(\Sigma)$ and satisfying the following requirements: i) holomorphy in $\mu$ :

$$
\frac{\delta \Gamma(\mu)}{\delta \bar{\mu}}=0
$$

ii) solution of the conformal Ward identity (1.16):

$$
s \Gamma(\mu)=\frac{1}{12 \pi} \int_{\Sigma} \frac{d \bar{z} \wedge d z}{2 i} C(\mu)\left(\partial^{3}+2 \mathscr{R}_{0} \partial+\left(\partial \mathscr{R}_{0}\right)\right) \mu
$$

(cf. Sect. 3). Here, $\mathscr{B}_{0}$ is a 0 -holomorphic projective connection on $\Sigma$ (cf. Sect. 2). $\mathscr{R}_{0}$ renders the integrand in (4.2) a well-defined density and ensures that the nilpotency condition (3.2) is satisfied. $\Gamma(\mu)$ depends on $\mathscr{B}_{0}$. Since $\mathscr{B}_{0}$ is fixed once for all, I shall not indicate explicitly such dependence. Next, I shall give the general expression of the Polyakov action and I shall try to motivate it.

Since $\Gamma(\mu)$ has well defined transformation properties under the action of the diffeomorphism group $\operatorname{Diff}_{c}(\Sigma)$, as follows from the Ward identity (4.2), it should be possible to define it in some open $\operatorname{Diff}_{c}(\Sigma)$-invariant subset $\odot$ of $\operatorname{Beltr}(\Sigma)$ (cf. Sect. 3).

The expression for $\Gamma(\mu)$ can be guessed to some extent through the following heuristic arguments. The original expression proposed by Polyakov for the planar topology is

$$
\Gamma(\mu)=-\frac{1}{24 \pi} \int_{\mathbf{C}} \mu \partial^{2} \ln \partial Z(\mu),
$$

where $Z(\mu)$ is a solution of the Beltrami equation (2.5) on $\mathbf{C}$ satisfying the asymptotic condition $Z(\mu)-z \rightarrow 0$ as $|z| \rightarrow \infty$ [16]. On a Riemann surface $\Sigma$, one expects the combination $\partial Z$ to be replaced by a holomorphic $\operatorname{Diff}_{c}(\Sigma)$-invariant section $\omega$ of $\mathscr{H}^{1}(\Sigma)$ on $(2$ (cf. Sects. 2 and 3) which is not identically zero for any $\mu$ in $(2$. This would yield an integrand of the form

$$
\mathscr{L}(\mu)=-\frac{1}{24 \pi} \mu \partial^{2} \ln \omega(\mu) .
$$


This object, however, is not a conformal field of weights 1,1 and hence cannot be integrated on $\Sigma$. To compensate for this shortcoming, one picks a non-identically zero element $\omega_{0}$ of $H^{1}(\Sigma, 0)$ (cf. Sect. 2) and replaces the above expression by the following one

$$
\mathscr{L}(\mu)=-\frac{1}{24 \pi} \mu\left(\partial-\left(\partial \ln \omega_{0}\right)\right) \partial \ln \left(\omega(\mu) / \omega_{0}\right) .
$$

This indeed is a conformal field of weights 1,1 but it is obviously not sufficient to build the Polyakov action. To yield the anomaly (4.2), there must be an additional contribution containing a term of the form $(1 / 24 \pi) 2 \mu \mathscr{R}_{0}$ as readily appears from (4.2) and (3.5). Such term is not in itself a conformal field of weights 1,1 and cannot be integrated on $\Sigma$. To correct for this, one subtracts a similar term $(1 / 24 \pi) 2 \mu \mathscr{R}_{0}^{\prime}$, where $\mathscr{B}_{0}^{\prime}$ is some $\mu$-independent projective connection. A natural choice is $\mathscr{B}_{0}^{\prime}=$ $\partial^{2} \ln \omega_{0}-\frac{1}{2}\left(\partial \ln \omega_{0}\right)^{2}$. This yields a further contribution to the integrand

$$
\mathscr{L}^{\prime}(\mu)=\frac{1}{24 \pi} 2 \mu\left[\mathscr{B}_{0}-\partial^{2} \ln \omega_{0}+\frac{1}{2}\left(\partial \ln \omega_{0}\right)^{2}\right] .
$$

On a general Riemann surface $\Sigma$, a third contribution of the form

$$
\mathscr{L}^{\prime \prime}(\mu)=\frac{1}{24 \pi}\left[\alpha \partial(\partial+(\partial \ln \omega(\mu))) \mu+\beta \partial\left(\partial+\left(\partial \ln \omega_{0}\right)\right) \mu\right]
$$

may be included, where $\alpha$ and $\beta$ are arbitrary complex constants. $\mathscr{L}^{\prime \prime}(\mu)$ is a total derivative. However, because of its singularities at the zeros of $\omega(\mu)$ and $\omega_{0}$, it gives a non-zero contribution upon integration on $\Sigma$. As it turns out, the correct choice of $\alpha$ and $\beta$ is $\alpha=\beta=1$. Summing up all the above contributions and performing some simple rearrangements, one obtains the first contribution to the Polyakov action:

$$
\begin{aligned}
\Gamma_{1}(\mu)= & \frac{1}{24 \pi} \int_{\Sigma} \frac{d \bar{z} \wedge d z}{2 i}\left[2 \mathscr{B}_{0} \mu+2 \partial^{2} \mu+\partial \ln \omega_{0} \partial \mu\right. \\
& \left.+\partial \ln \omega(\mu) \partial \mu+\partial \ln \omega_{0} \partial \ln \omega(\mu) \mu\right] .
\end{aligned}
$$

For genus 1 , this object would indeed be a continuous functional of $\mu$ satisfying (4.1)-(4.2). Formal manipulations would seem to suggest that this is true also in higher genera but a careful analysis shows that this cannot be so, as I shall now explain. Recall that, for any Beltrami differential $\mu$ in $\operatorname{Beltr}(\Sigma)$, every monodromic $\mu$-holomorphic 1 -differential has precisely $2 g-2$ zeros counting multiplicity [28]. For $\mu$ in $\mathscr{Q}$, denote by $P_{j}(\mu)$ the distinct zeros of $\omega(\mu)$. Similarly, denote by $P_{0 k}$ the distinct zeros of $\omega_{0}$. Finally, denote by $\mathcal{O}^{\prime}$ the set of all $\mu$ 's in $(2)$ such that no one of the $P_{\jmath}(\mu)$ 's coincide with any of the $P_{0 k}$ 's. $\mathscr{Q}^{\prime}$ is a non-empty open subset of $\mathscr{Q}$. The integrand of (4.4) is clearly singular at the $P_{j}(\mu)$ 's and $P_{0 k}$ 's. It is absolutely integrable for any $\mu$ in $\mathcal{C}^{\prime}$, but only conditionally convergent for $\mu$ in $\left(O^{\prime}\right.$. It follows from here that $\Gamma_{1}(\mu)$ is continuous for $\mu$ varying in $\mathscr{Q}^{\prime}$ while it is not at the $\mu$ 's lying in $\mathcal{O}^{\prime} \mathcal{O}^{\prime}$. Further, when varying $\Gamma_{1}(\mu)$ with respect to $\mu, \bar{\mu}$, it is not possible to straightforwardly interchange integration and variation, since the variation of the integrand produces absolutely non-integrable singularities at the zeros $P_{j}(\mu)$ of $\omega(\mu)$. Upon employing a cut-off procedure, one verifies that $\Gamma_{1}(\mu)$ does not fulfill (4.1)-(4.2).

One is thus forced to add to $\Gamma_{1}(\mu)$ a term $\Gamma_{2}(\mu)$ compensating for the above diseases. The nature of $\Gamma_{2}(\mu)$ is determined by the requirement that the combination 
$\Gamma_{1}(\mu)+\Gamma_{2}(\mu)$ is singlevalued and continuous and satisfies (4.1)-(4.2) on $\odot$. A direct analysis shows that $\Gamma_{2}(\mu)$ is given by an expression of the form

$$
\Gamma_{2}(\mu)=\frac{1}{24}\left[\sum_{j} \nu_{j}(\mu) \ln \left(\Omega(\mu) / \omega_{0}\right)\left(\widehat{P}_{\jmath}(\mu)\right)+\sum_{k} \nu_{0 k} \ln \left(\omega(\mu) / \Omega_{0}\right)\left(\widehat{P}_{0 k}\right)\right] .
$$

Here, $\Omega$ is a holomorphic $\operatorname{Diff}_{c}(\Sigma)$-invariant section of $\mathscr{H}^{1}(\Sigma, \chi)$ on $\mathscr{O}$, where $\chi$ is a holomorphic Diff $c(\Sigma)$-invariant section of the multiplier bundle $\mathscr{G}_{\pi}$ on $(2$ (cf. Sects. 2 and 3). It is further assumed that, for any $\mu$ in $\mathscr{Q}, \Omega(\mu)$ is nowhere vanishing. Similarly, $\Omega_{0}$ is a nowhere vanishing element of $H^{1}\left(\Sigma, \chi_{0}, 0\right)$, where $\chi_{0}$ is a 0-holomorphic multiplier (cf. Sect. 2). $\nu_{j}(\mu)$ is the order of the zero $P_{j}(\mu)$ of $\omega(\mu)$. Similarly, $\nu_{0 k}$ is the order of the zero $P_{0 k}$ of $\omega_{0} . \widehat{P}_{j}(\mu)$ is any chosen lift of $P_{j}(\mu)$ to the universal covering surface $\Sigma_{\pi}$ of the universal covering $\pi$ depending continuously on $\mu$ (cf. Sect. 2). Likewise, $\widehat{P}_{0 k}$ is a fixed lift of $P_{0 k}$ to $\Sigma_{\pi}$. The appearance of polydromic fields may seem intriguing and requires an explanation. If $\Omega(\mu)$ had zeros, then $\Gamma_{1}(\mu)+\Gamma_{2}(\mu)$ would still define a local solution in $\mathscr{O}$ of (4.1)-(4.2). But $\Gamma_{2}(\mu)$ would be singular at those $\mu$ 's for which some of the $\widehat{P}_{j}(\mu)$ 's are zeros of $\Omega(\mu)$. Further, it may happen that when $\mu$ is transported along a closed loop, some of the $P_{j}(\mu)$ 's circle around some of the zeros of $\Omega(\mu)$. This would render $\Gamma_{2}(\mu)$ multivalued in $\mathscr{G}$, a disease that one would like to avoid. If one assumes that $\Omega(\mu)$ is nowhere vanishing, then $\Omega$ is necessarily polydromic. In fact, as noticed earlier, monodromic $\mu$-holomorphic 1-differentials necessarily have zeros [28]. Only polydromic $\mu$-holomorphic 1-differentials can be free of zeros. See Eq. (4.70) below for a concrete example. The assumption that $\Omega_{0}$ is free of zeros is, conversely, merely simplifying. $\Gamma_{2}(\mu)$ obviously depends on the choice of the lifts $\widehat{P}_{j}(\mu)$ and $\widehat{P}_{0 k}$ of the $P_{j}(\mu)$ 's and the $P_{0 k}$ 's.

It will be shown below that the sum $\Gamma_{1}(\mu)+\Gamma_{2}(\mu)$ has the nice properties stated above. One reaches the conclusion that the most general Polyakov action $\Gamma(\mu)$ on $($ ) can be written as

$$
\Gamma(\mu)=\Gamma_{1}(\mu)+\Gamma_{2}(\mu)+\Phi(\mu)
$$

where $\Gamma_{1}(\mu)$ and $\Gamma_{2}(\mu)$ are given respectively by (4.4) and (4.5) and $\Phi(\mu)$ is a continuous functional of $\mu$ in $\odot$ such that

$$
\begin{gathered}
\frac{\delta \Phi(\mu)}{\delta \bar{\mu}}=0, \\
s \Phi(\mu)=0 .
\end{gathered}
$$

Unlike what happens for the planar geometry, the functional $\Phi(\mu)$ cannot be determined from first principles. $\Phi(\mu)$ carries all the model dependent features of conformal field theory on $\Sigma$.

In ref. [25], an alternative expression of $\Gamma(\mu)$ was provided. It can be shown that that expression is indeed equivalent to (4.6). The proof is based on a simple algebraic identity following from (2.15) and Stokes' theorem. Details are not provided for the sake of brevity.

Let us now proceed to the demonstration of the above statements. For brevity, I shall not indicate explicitly the dependence on $\mu$ of the various objects. Before entering into the technical details of the proofs, a few preliminary results and remarks are in order. 
i) The Generalized Cauchy Residue Formula. The following mathematical lemma will be used repeatedly below. Let $f$ be a function defined and smooth near a point $P$ of $\Sigma$ and $Z$ a coordinate of $\mathbf{A}(\mu)$ defined at $P$. Then, for any integer $m \geq 0$, one has

$$
\lim _{\varepsilon \rightarrow 0+} \frac{1}{2 \pi i} \oint_{\partial B(P, \varepsilon)} \frac{d Z}{(Z-Z(P))^{m+1}} f=\frac{1}{m !}\left(\frac{1}{\partial Z} \partial\right)^{m} f(P),
$$

where $B(P, \varepsilon)$ is the disk $\left\{P^{\prime}: P^{\prime} \in \Sigma,\left|z\left(P^{\prime}\right)-z(P)\right|<\varepsilon\right\}, z$ being any coordinate of a defined at $P$. To prove this identity, one first computes the left-hand side of (4.7) for $f=\overline{(Z-Z(P))}^{n}$ with $0 \leq n \leq m$. The calculation is carried out by expanding $Z$ in powers of $z-z(P)$ and $\overline{z-z(P)}$ and keeping only the terms of order $\varepsilon^{-k}$ with non-negative $k$. The contour integration and the limit can be performed by means of standard techniques of complex calculus. At this stage, it is crucial to use the bound $|\mu|<1$. In this way, one verifies (4.7) for the above special choice of $f$. The result generalizes to an arbitrary function $f$ by expanding $f$ in powers of $Z-Z(P)$ and $\overline{Z-Z(P)}$ to order $m$.

ii) Choice of Local Coordinates at the $P_{j}(\mu)^{\prime}$ 's and the $P_{0 k}$ 's. In the following analysis, I shall employ a special set of coordinates. Such local parameters will be used throughout, but the final result will be independent from their choice. Let $Z_{j}$ be a coordinate of $\mathbf{A}(\mu)$ defined near $P_{j}$ and normalized so that $Z_{j}\left(P_{j}\right)=0$ identically in $\mu$. Similarly, let $Z_{0 k}$ be a coordinate of $\mathbf{A}(0)$ defined near $P_{0 k}$ and normalized so that $Z_{0 k}\left(P_{0 k}\right)=0$. Since $\omega$ has a zero of order $\nu_{j}$ at $P_{j}$, one has the singular expansion

$$
\ln \omega=\nu_{j} \ln Z_{j}+\text { regular terms }
$$

near $P_{j}$ and, analogously,

$$
\ln \omega_{0}=\nu_{0 k} \ln Z_{0 k}+\text { regular terms }
$$

near $P_{0 k}$.

iii) Local versus Global Properties. For small variations of $\mu$, each $\widehat{P}_{j}(\mu)$ remains inside a given fundamental domain $D_{\text {j }}$ of $\pi$. An analogous statement holds also for each $\widehat{P}_{0 k}$ trivially. Recall that, for any fundamental domain $D$ of $\pi$, the restriction $\pi \mid D$ of $\pi$ to $D$ is a biholomorphism when $\Sigma$ carries the reference conformal structure a and $\Sigma_{\pi}$ the lift by $\pi$ of a (cf. Sect. 2). Hence, in the analysis of the local properties of the relevant functionals in $\left(\right.$, one can act as if the polydromic fields $\Omega$ and $\Omega_{0}$ were defined in $\Sigma$ and identify $\widehat{P}_{j}$ and $\widehat{P}_{0 k}$ with $P_{j}$ and $P_{0 k}$, respectively. This will no longer be possible when analyzing the global properties of those functionals in 0

After the above preliminaries, one may proceed to the proof of the key properties of $\Gamma(\mu)$.

i) $\Gamma(\mu)$ is well-defined for $\mu$ in $\mathcal{O}^{\prime}$.

Proof. The proof that the integrand in expression (4.4) is a well-defined density is straightforward. From (4.8)-(4.9), it follows readily that, for $\mu$ in $0^{\prime}$, the integrand diverges as $\sim 1 / Z_{j}$ near $P_{j}$ and as $\sim 1 / Z_{0 k}$ near $P_{0 k}$. Thus, it is absolutely integrable. The well-definedness of the expression (4.5) for $\mu$ in $G^{\prime \prime}$ is manifest. QED

ii) By a suitable choice of the branch of the logarithms, it is possible to render $\Gamma(\mu)$ continuous on any sufficiently small neighborhood in $G^{\prime \prime}$.

Proof. Since the integrand density in the expression (4.4) of $\Gamma_{1}$ is absolutely integrable for $\mu$ in $\mathscr{G}^{\prime}$ and depends continuously on $\mu$ in that range and the integration domain 
is compact, $\Gamma_{1}$ is continuous in $\mathscr{G}^{\prime}$. By inspection, it is apparent that, by a suitable choice of the branch of the logarithms in expression (4.5), $\Gamma_{2}$ can be made continuous on any sufficiently small open subset of $\mathcal{C}^{\prime}$. Clearly, this latter property holds also for the sum $\Gamma_{1}+\Gamma_{2}$. QED

iii) Calculation of the variations $\delta \Gamma(\mu)$ and $\bar{\delta} \Gamma(\mu)$ in $\mathcal{O}^{\prime}$. Here, $\delta(\bar{\delta})$ denotes infinitesimal variation with respect to $\mu(\bar{\mu})$. The calculation is required for those of $\delta \Gamma(\mu) / \delta \mu$, $\delta \Gamma(\mu) / \bar{\delta} \mu$ and $s \Gamma(\mu)$, which in turn are necessary for obtaining the expression of the energy momentum tensor and for showing (4.1) and (4.2).

Proof. The derivation of the basic variational formulae for $\Gamma$ invokes repeatedly the following relations which follow from the definition of $\omega, \Omega, \omega_{0}$ and $\Omega_{0}$ and from (2.15) with $j=1$ perhaps with $\mu=0$ and from (2.12) with $\mu=0$ :

$$
\begin{gathered}
(\bar{\partial}-\mu \partial) \ln \omega-\partial \mu=0, \\
(\bar{\partial}-\mu \partial) \ln \Omega-\partial \mu=0, \\
\bar{\partial} \ln \omega_{0}=0, \\
\bar{\partial} \ln \Omega_{0}=0, \\
\bar{\partial} \mathscr{R}_{0}=0 .
\end{gathered}
$$

Then

$$
\begin{aligned}
(\bar{\partial}-\mu \partial) \delta \ln \omega-(\partial+(\partial \ln \omega)) \delta \mu=0, & \bar{\delta} \ln \omega=0, \\
(\bar{\partial}-\mu \partial) \delta \ln \Omega-(\partial+(\partial \ln \Omega)) \delta \mu=0, & \bar{\delta} \ln \Omega=0, \\
\delta \ln \omega_{0}=0, & \bar{\delta} \ln \omega_{0}=0, \\
\delta \ln \Omega_{0}=0, & \bar{\delta} \ln \Omega_{0}=0, \\
\delta \mathscr{R}_{0}=0, & \bar{\delta} \mathscr{R}_{0}=0 .
\end{aligned}
$$

Relations (4.15a) and (4.16a) follow readily from (4.10) and (4.11), respectively. Equations (4.15b) and (4.16b) are a direct consequence of the holomorphy in $\mu$ of $\omega$ and $\Omega$, respectively. (4.17)-(4.19) follow trivially from the independence of $\omega_{0}, \Omega_{0}$ and $\mathscr{R}_{0}$ from $\mu$.

Let us compute the variation $\delta \Gamma$. Suppose that $\mu$ lies in $\mathscr{Q}^{\prime}$, so that no one of the $P_{j}$ 's coincide with any of the $P_{0 k}$ 's. Consider first $\Gamma_{1}$. As already indicated at the beginning of this section, in the computation of $\delta \Gamma_{1}$, it is not possible to straightforwardly interchange the order of integration and variation when applying $\delta$ to the right-hand side of (4.4). Indeed, from (4.8)-(4.9), it is apparent that the application of $\delta$ to the combination $\partial \ln \omega$ produces an absolutely non-integrable singularity $\sim-\nu_{\jmath} \partial Z_{j} \delta Z_{j} / Z_{j}^{2}$ near $P_{j}$. This mandates the introduction of a cutoff. Choose points $Q_{j}$ of $\Sigma$ independent from $\mu$ and $\varepsilon_{j}>0$ such that $P_{j}$ is contained in $B\left(Q_{\jmath}, \varepsilon_{j}\right)$ and $B\left(Q_{j}, \varepsilon_{j}\right)$ and $B\left(Q_{\jmath^{\prime}}, \varepsilon_{j^{\prime}}\right)$ have empty intersection for $j \neq j^{\prime}$. In (4.4), the integral over $\Sigma$ is the sum of an integral over $\Sigma \backslash \cup_{j} B\left(Q_{j}, \varepsilon_{j}\right)$ and one over $\cup_{j} B\left(Q_{\jmath}, \varepsilon_{\jmath}\right)$. Upon applying the variation $\delta$, one can interchange the order of integration and variation in the first integral, since the integration domain is independent from $\mu$ and the integration singularities produced by the action of $\delta$ on the integrand are well outside of the integration domain. The action of $\delta$ on the second integral can be dealt with by exploiting the fact that the integrand is near $P_{j}$ of the form $\left(\partial+\left(\partial \ln \omega_{0}\right)\right) \mu \nu_{j} \partial Z_{j} / Z_{j}+$ terms that are regular when acted upon by 
$\delta$, by (4.8)-(4.9). Since the variation cannot depend on the choice of the $Q_{j}$ 's and $\varepsilon_{j}$ 's one can set $Q_{j}=P_{j}$ and take the limit $\varepsilon_{j} \rightarrow 0+$ at the very end to simplify the resulting expression. Proceeding in this way, one obtains

$$
\delta \Gamma_{1}=\delta_{a} \Gamma+\delta R
$$

where

$$
\begin{aligned}
\delta_{a} \Gamma= & \frac{1}{24 \pi} \lim _{\varepsilon_{\jmath} \rightarrow 0+} \int_{\Sigma \backslash \cup_{\jmath} B\left(P_{\jmath}, \varepsilon_{j}\right)} \frac{d \bar{z} \wedge d z}{2 i} \delta\left[2 \mathscr{R}_{0} \mu+2 \partial^{2} \mu+\partial \ln \omega_{0} \partial \mu\right. \\
& \left.+\partial \ln \omega \partial \mu+\partial \ln \omega_{0} \partial \ln \omega \mu\right] \\
\delta R= & \frac{1}{24 \pi} \sum_{j} \nu_{\jmath} \lim _{\varepsilon_{\jmath} \rightarrow 0+}\left\{\delta \int_{B\left(Q_{j}, \varepsilon_{\jmath}\right)} \frac{d \bar{z} \wedge d z}{2 i} \frac{\left(\partial+\left(\partial \ln \omega_{0}\right)\right) \mu \partial Z_{j}}{Z_{\jmath}}\right\}_{Q_{j}=P_{\jmath}}
\end{aligned}
$$

The existence and finiteness of the limit as the $\varepsilon_{j}$ 's tend to 0 of the surface integral in the right-hand side of (4.21) follow from standard analytic arguments from the theory of Cauchy principal value and from the fact that $|\mu|<1$ everywhere in $\Sigma$. The existence and finiteness of the limit as the $\varepsilon_{j}$ 's tend to 0 of the expression in the right-hand side of (4.22) will be shown in a moment. Consider $\Gamma_{2}$. The calculation of $\delta \Gamma_{2}$ is straightforward from (4.5), (4.11)-(4.12). The result has the following form

$$
\delta \Gamma_{2}=\delta_{b} \Gamma+\delta S
$$

where

$$
\begin{gathered}
\delta_{b} \Gamma=\frac{1}{24}\left\{\sum_{j} \nu_{\jmath}\left[\delta \ln \Omega\left(P_{j}\right)-\frac{\partial \ln \left(\Omega / \omega_{0}\right)}{\partial Z_{\jmath}} \delta Z_{j}\left(P_{j}\right)\right]+\sum_{k} \nu_{0 k} \delta \ln \omega\left(P_{0 k}\right)\right\} \\
\delta S=\frac{1}{24} \sum_{j} \nu_{j}\left[\left(\partial+\left(\partial \ln \omega_{0}\right)\right) \mu\left(P_{j}\right) \delta \bar{z}\left(P_{\jmath}\right)+\frac{\partial \ln \left(\Omega / \omega_{0}\right)}{\partial Z_{\jmath}}\left(P_{j}\right)\left(\delta Z_{j}\left(P_{\jmath}\right)\right.\right. \\
\left.\left.+\left(\delta z\left(P_{j}\right)+\mu\left(P_{\jmath}\right) \delta \bar{z}\left(P_{j}\right)\right) \partial Z_{j}\left(P_{j}\right)\right)\right]
\end{gathered}
$$

Now, I shall show that $\delta R=-\delta S$. To this end, a few preliminary identities are needed. By applying the variation $\delta$ to the identity $Z_{j}\left(P_{\jmath}\right)=0$ and using (2.5), one obtains

$$
\delta Z_{j}\left(P_{j}\right)+\left(\delta z\left(P_{j}\right)+\mu\left(P_{j}\right) \delta \bar{z}\left(P_{\jmath}\right)\right) \partial Z_{\jmath}\left(P_{\jmath}\right)=0
$$

Hence, the second term within square brackets in (4.25) vanishes identically. Next, from (2.5), one has the variational identity $(\bar{\partial}-\mu \partial) \delta Z_{j}=\delta \mu \partial Z_{j}$. From this relation 
and (4.7), one finds that, for any function $f$ defined and smooth at $P_{\jmath}$,

$$
\begin{aligned}
& \lim _{\varepsilon \rightarrow 0+} \frac{1}{2 \pi i} \oint_{\partial B\left(P_{j}, \varepsilon_{j}\right)} \frac{d \bar{z} \delta \mu \partial Z_{j}}{Z_{j}} f \\
& \quad=f\left(P_{j}\right) \lim _{\varepsilon_{j} \rightarrow 0+} \frac{1}{2 \pi i} \oint_{\partial B\left(P_{\jmath}, \varepsilon_{j}\right)}\left\{d\left(\frac{\delta Z_{j}}{Z_{j}}\right)+\frac{d Z_{\jmath}}{Z_{\jmath}^{2}} \delta Z_{j}-\frac{d Z_{\jmath}}{Z_{\jmath}} \frac{\partial \delta Z_{j}}{\partial Z_{j}}\right\} \\
& =0
\end{aligned}
$$

Next, from (2.5) and (4.12), one has the identity

$$
\begin{aligned}
(\partial+ & \left.\left(\partial \ln \omega_{0}\right)\right) \mu \partial Z_{j} / Z_{j} \\
& =(\bar{\partial}-\mu \partial) \ln \left(\partial Z_{\jmath} / \omega_{0}\right) \partial Z_{j} / Z_{j} \\
& =\bar{\partial}\left[\ln \left(\partial Z_{\jmath} / \omega_{0}\right) \partial Z_{j} / Z_{j}\right]-\partial\left[\mu \ln \left(\partial Z_{\jmath} / \omega_{0}\right) \partial Z_{j} / Z_{j}\right] .
\end{aligned}
$$

From (4.28), Stokes' theorem, (2.8), (4.7), (4.26), (4.27) and the variational identity $\delta d Z_{\jmath}=d Z_{j} \partial \delta Z_{j} / \partial Z_{j}+d \bar{z} \delta \mu \partial Z_{j}$, which follows readily from (2.8), one gets

$$
\begin{aligned}
\lim _{\varepsilon_{j} \rightarrow 0+}\left\{\delta \int_{B\left(Q_{\jmath}, \varepsilon_{\jmath}\right)} \frac{d \bar{z} \wedge d z}{2 i} \frac{\left(\partial+\left(\partial \ln \omega_{0}\right)\right) \mu \partial Z_{j}}{Z_{j}}\right\}_{Q_{j}=P_{\jmath}} \\
=\pi \lim _{\varepsilon_{j} \rightarrow 0+}\left\{\frac{1}{2 \pi i} \delta \oint_{\partial B\left(Q_{\jmath}, \varepsilon_{j}\right)} \frac{d Z_{j}}{Z_{\jmath}} \ln \left(\partial Z_{\jmath} / \omega_{0}\right)\right. \\
\left.\quad-\delta \lim _{\varrho_{j} \rightarrow 0+} \frac{1}{2 \pi i} \oint_{\partial B\left(P_{\jmath}, \varrho_{\jmath}\right)} \frac{d Z_{j}}{Z_{\jmath}} \ln \left(\partial Z_{j} / \omega_{0}\right)\right\}_{Q_{\jmath}=P_{j}} \\
=\pi \lim _{\varepsilon_{\jmath} \rightarrow 0+} \frac{1}{2 \pi i} \oint_{\partial B\left(P_{j}, \varepsilon_{j}\right)}\left\{\frac{d Z_{j}}{Z_{j}^{2}}\left[-\delta Z_{j} \ln \left(\partial Z_{\jmath} / \omega_{0}\right)\right]+\frac{d Z_{\jmath}}{Z_{j}}\left[\delta \ln \left(\partial Z_{j} / \omega_{0}\right)\right.\right. \\
\left.\quad+\frac{\ln \left(\partial Z_{j} / \omega_{0}\right) \partial \delta Z_{j}}{\partial Z_{\jmath}}+\frac{d \bar{z} \partial Z_{\jmath} \delta \mu}{Z_{j}} \ln \left(\partial Z_{\jmath} / \omega_{0}\right)\right\}-\pi \delta\left[\ln \left(\partial Z_{j} / \omega_{0}\right)\left(P_{j}\right)\right] \\
=-\pi\left(\partial+\left(\partial \ln \omega_{0}\right)\right) \mu\left(P_{\jmath}\right) \delta \bar{z}\left(P_{j}\right) .
\end{aligned}
$$

From (4.22), (4.25), (4.26), and (4.29), it follows immediately that $\delta R+\delta S=0$, as announced earlier. Then form (4.6a), (4.20), and (4.23), one finds the relation

$$
\delta \Gamma=\delta_{a} \Gamma+\delta_{b} \Gamma+\delta \Phi,
$$

where $\delta_{a} \Gamma$ and $\delta_{b} \Gamma$ are given by (4.21) and (4.24), respectively. Let us compute $\delta_{a} \Gamma$. Consider the integrand in the right-hand side of (4.21). By (4.17a) and (4.19a), one finds

$$
\begin{aligned}
& \delta\left[2 \mathscr{R}_{0} \mu+2 \partial^{2} \mu+\partial \ln \omega_{0} \partial \mu+\partial \ln \omega \partial \mu+\partial \ln \omega_{0} \partial \ln \omega \mu\right] \\
&= \partial\left[(\partial+(\partial \ln \omega)) \delta \mu+\left(\partial+\left(\partial \ln \omega_{0}\right)\right) \delta \mu+\left(\partial+\left(\partial \ln \omega_{0}\right)\right) \mu \delta \ln \omega\right] \\
&-\partial\left(\partial+\left(\partial \ln \omega_{0}\right)\right) \mu \delta \ln \omega \\
&+\left[2 \mathscr{B}_{0}-2 \partial^{2} \ln \omega_{0}+\left(\partial \ln \omega_{0}\right)^{2}-\left(\partial-\left(\partial \ln \omega_{0}\right)\right) \partial \ln \left(\omega / \omega_{0}\right)\right] \delta \mu .
\end{aligned}
$$


From (4.10), (4.12) and (4.15a), one has

$$
\begin{aligned}
- & \partial\left(\partial+\left(\partial \ln \omega_{0}\right)\right) \mu \delta \ln \omega \\
= & -(\bar{\partial}-\mu \partial-(\partial \mu)) \partial \ln \left(\omega / \omega_{0}\right) \delta \ln \omega \\
= & \partial\left[\mu \partial \ln \left(\omega / \omega_{0}\right) \delta \ln \omega\right]-\bar{\partial}\left[\partial \ln \left(\omega / \omega_{0}\right) \delta \ln \omega\right]+\partial \ln \left(\omega / \omega_{0}\right)(\bar{\partial}-\mu \partial) \delta \ln \omega \\
= & \partial\left[\partial \ln \left(\omega / \omega_{0}\right) \delta \mu+\mu \partial \ln \left(\omega / \omega_{0}\right) \delta \ln \omega\right]-\bar{\partial}\left[\partial \ln \left(\omega / \omega_{0}\right) \delta \ln \omega\right] \\
& -\left[\left(\partial-\left(\partial \ln \omega_{0}\right)\right) \partial \ln \left(\omega / \omega_{0}\right)-\left(\partial \ln \left(\omega / \omega_{0}\right)\right)^{2}\right] \delta \mu .
\end{aligned}
$$

From (4.10), (4.12), one has further

$$
(\partial+(\partial \ln \omega)) \mu=\bar{\partial} \ln \left(\omega / \omega_{0}\right) .
$$

By introducing (4.32) into (4.31) and then by using (4.33), one obtains

$$
\begin{aligned}
\delta\left[2 \mathscr{R}_{0} \mu+2 \partial^{2} \mu+\partial \ln \omega_{0} \partial \mu+\partial \ln \omega \partial \mu+\partial \ln \omega_{0} \partial \ln \omega \mu\right] \\
=\partial[2(\partial+(\partial \ln \omega)) \delta \mu \\
\left.+\bar{\partial} \ln \left(\omega / \omega_{0}\right) \delta \ln \omega\right]-\bar{\partial}\left[\partial \ln \left(\omega / \omega_{0}\right) \delta \ln \omega\right] \\
\quad+\left[2 \mathscr{R}_{0}-2 \partial^{2} \ln \omega+(\partial \ln \omega)^{2}\right] \delta \mu .
\end{aligned}
$$

Next, one introduces (4.34) into (4.21). The total derivative terms yield, upon applying Stokes' theorem, a set of line integrals on loops of infinitesimal size cut around the singularities of those terms, that is the $P_{j}$ 's and the $P_{0 k}$ 's. Let us compute those line integrals. From the singular expansion (4.8) and from (4.27), one finds

$$
\begin{aligned}
\lim _{\varepsilon_{j} \rightarrow 0+} \frac{1}{2 \pi i} \oint_{\partial B\left(P_{\jmath}, \varepsilon_{\jmath}\right)} d \bar{z}(\partial+(\partial \ln \omega)) \delta \mu & =\lim _{\varepsilon_{\jmath} \rightarrow 0+} \frac{1}{2 \pi i} \oint_{\partial B\left(P_{j}, \varepsilon_{\jmath}\right)} \frac{d \bar{z} \delta \mu \partial Z_{\jmath}}{Z_{j}} \nu_{\jmath} \\
& =0 .
\end{aligned}
$$

Next, set

$$
f_{j}\left(Z_{j}\right)=\omega /\left(\partial Z_{j} Z_{\jmath}^{\nu_{\jmath}}\right)
$$

locally in the domain of $Z_{j}$. From (2.5) and (2.15) with $j=1$, it follows readily that $f_{j}(\zeta)$ is a holomorphic function defined and non-vanishing at $\zeta=0$. From (4.36) and (4.7), one obtains

$$
\begin{aligned}
\lim _{\varepsilon_{\jmath} \rightarrow 0+} & \frac{1}{2 \pi i} \oint_{\partial B\left(P_{j}, \varepsilon_{j}\right)} d \ln \left(\omega / \omega_{0}\right) \delta \ln \omega \\
= & \lim _{\varepsilon_{j} \rightarrow 0+} \frac{1}{2 \pi i} \oint_{\partial B\left(P_{\jmath}, \varepsilon_{j}\right)}\left\{\frac{d Z_{j}}{Z_{j}^{2}} \nu_{j}^{2} \delta Z_{\jmath}+\frac{d Z_{j}}{Z_{j}} \nu_{j}\left[\frac{\partial \delta Z_{\jmath}}{\partial Z_{j}}\right.\right. \\
& \left.\left.+\frac{2 \partial_{\zeta} f_{j}\left(Z_{j}\right) \delta Z_{j}}{f_{j}\left(Z_{j}\right)}+\frac{\delta f_{j}\left(Z_{j}\right)}{f_{\jmath}\left(Z_{j}\right)}\right]+d \ln \left(\partial Z_{\jmath} / \omega_{0}\right) \frac{\nu_{j} \delta Z_{\jmath}}{Z_{\jmath}}\right\} \\
= & \nu_{j}\left[\left(\nu_{j}+1\right) \frac{\partial \delta Z_{j}}{\partial Z_{j}}\left(P_{j}\right)+\frac{2 \partial_{\zeta} f_{j}(0)}{f_{j}(0)} \delta Z_{j}\left(P_{\jmath}\right)+\frac{\delta f_{\jmath}(0)}{f_{j}(0)}\right. \\
& \left.+\frac{\partial \ln \left(\partial Z_{j} / \omega_{0}\right) \delta Z_{\jmath}}{Z_{j}}\left(P_{j}\right)\right] .
\end{aligned}
$$


From the singular expansion (4.9) and from (4.7), one finds

$$
\begin{aligned}
\lim _{\varepsilon_{0 k} \rightarrow 0+} \frac{1}{2 \pi i} \oint_{\partial B\left(P_{0 k}, \varepsilon_{0 k}\right)} d \ln \left(\omega / \omega_{0}\right) \delta \ln \omega \\
\quad=\lim _{\varepsilon_{0 k} \rightarrow 0+} \frac{1}{2 \pi i} \oint_{\partial B\left(P_{0 k}, \varepsilon_{0 k}\right)} \frac{d Z_{0 k}}{Z_{0 k}}\left(-\nu_{0 k} \delta \ln \omega\right) \\
=-\nu_{0 k} \delta \ln \omega\left(P_{0 k}\right)
\end{aligned}
$$

From (4.21), (4.34), Stokes' theorem, (4.35)-(4.38), one finds eventually

$$
\begin{aligned}
\delta_{a} \Gamma= & \frac{1}{12 \pi} \lim _{\varepsilon_{j} \rightarrow 0+} \int_{\Sigma \backslash \cup_{j} B\left(P_{\jmath}, \varepsilon_{\jmath}\right)} \frac{d \bar{z} \wedge d z}{2 i} \delta \mu\left[\mathscr{R}_{0}-\partial^{2} \ln \omega+\frac{1}{2}(\partial \ln \omega)^{2}\right] \\
& +\frac{1}{24}\left\{\sum _ { j } \nu _ { j } \left[\left(\nu_{j}+1\right) \frac{\partial \delta Z_{j}}{\partial Z_{j}}\left(P_{j}\right)+\frac{2 \partial_{\zeta} f_{j}(0)}{f_{j}(0)} \delta Z_{j}\left(P_{j}\right)+\frac{\delta f_{j}(0)}{f_{j}(0)}\right.\right. \\
& \left.\left.+\frac{\partial \ln \left(\partial Z_{j} / \omega_{0}\right) \delta Z_{\jmath}}{\partial Z_{j}}\left(P_{\jmath}\right)\right]-\sum_{k} \nu_{0 k} \delta \ln \omega\left(P_{0 k}\right)\right\} .
\end{aligned}
$$

The computation of $\delta_{b} \Gamma$ is easier. To this end, define, locally in the domain of $Z_{j}$,

$$
F_{j}\left(Z_{j}\right)=\Omega / \partial Z_{j}
$$

From (2.5) and (2.15) with $j=1$, it follows readily that the function $F_{j}(\zeta)$ is holomorphic and non-vanishing at $\zeta=0$. By using (4.11), (4.12), (4.17a), (4.18a), and (4.40), one finds readily

$$
\begin{aligned}
\delta_{b} \Gamma= & \frac{1}{24}\left\{\sum _ { \jmath } \nu _ { j } \left[\frac{\partial \delta Z_{j}}{\partial Z_{j}}\left(P_{\jmath}\right)+\frac{\partial_{\zeta} F_{j}(0)}{F_{j}(0)} \delta Z_{\jmath}\left(P_{\jmath}\right)+\frac{\delta F_{j}(0)}{F_{j}(0)}\right.\right. \\
& \left.\left.-\frac{\partial \ln \left(\Omega / \omega_{0}\right) \delta Z_{j}}{\partial Z_{j}}\left(P_{j}\right)\right]+\sum_{k} \nu_{0 k} \delta \ln \omega\left(P_{0 k}\right)\right\}
\end{aligned}
$$

From (4.30), (4.39) and (4.41), one can easily compute $\delta \Gamma$. The result is

$$
\begin{gathered}
\delta \Gamma=\frac{1}{12 \pi} \lim _{\varepsilon_{j} \rightarrow 0+} \int_{\Sigma \backslash \cup_{j} B\left(p_{j}, \varepsilon_{j}\right)} \frac{d \bar{z} \wedge d z}{2 i} \delta \mu\left[\mathscr{B}_{0}-\partial^{2} \ln \omega+\frac{1}{2}(\partial \ln \omega)^{2}\right]+\delta \Phi \\
+\frac{1}{24} \sum_{j} \nu_{j}\left[\left(\nu_{j}+2\right) \frac{\partial \delta Z_{j}}{\partial Z_{j}}\left(P_{j}\right)+\frac{2 \partial_{\zeta} f_{j}(0)}{f_{j}(0)} \delta Z_{j}\left(P_{j}\right)\right. \\
\left.+\frac{\delta f_{j}(0)}{f_{j}(0)}+\frac{\delta F_{j}(0)}{F_{j}(0)}\right]
\end{gathered}
$$

(cf. (4.36) and (4.40)).

Let us compute $\bar{\delta} \Gamma$. Assume as before that $\mu$ lies in $\mathscr{O}^{\prime}$, so that no one of $P_{j}$ 's coincide with any of the $P_{0 k}$ 's. By a calculation completely analogous to that leading to (4.30), one can show that formulae similar to (4.21), (4.24) and (4.30) hold with the variational operator $\delta$ replaced everywhere by $\bar{\delta}$. To compute $\bar{\delta} \Gamma$, one needs to know 
$\bar{\delta} Z_{j}\left(P_{j}\right)$. It is not obvious that this vanishes, because the normalization condition $Z_{j}\left(P_{j}\right)=0$ may cause a non-holomorphic dependence on $\mu$, but it is nevertheless true. Since $P_{j}$ is a zero of $\omega$ of order $\nu_{\jmath}$, one has $\partial^{\nu_{\jmath}-1} \omega\left(P_{j}\right)=0$ but $\partial^{\nu_{\jmath}}\left(P_{j}\right) \neq 0$. By applying $\bar{\delta}$ to the former relation and taking into account the latter and (4.15b), one finds

$$
\bar{\delta} z\left(P_{j}\right)+\mu\left(P_{j}\right) \bar{\delta} \bar{z}\left(P_{j}\right)=0 .
$$

Now, by applying the variation $\bar{\delta}$ to the identity $Z_{j}\left(P_{j}\right)=0$, one obtains a relation similar to (4.26) with $\delta$ replaced by $\bar{\delta}$. From such relation and (4.43), one obtains

$$
\bar{\delta} Z_{j}\left(P_{j}\right)=0
$$

as announced. From (4.15b), (4.16b), (4.17b), (4.18b), (4.19b), (4.44), and the $\bar{\delta}$ analogs of (4.21), (4.24), and (4.30), one obtains immediately

$$
\bar{\delta} \Gamma=0
$$

This completes the calculation of the variations $\delta \Gamma$ and $\bar{\delta} \Gamma$. QED

iv) $\Gamma(\mu)$ is a continuous and singlevalued functional of $\mu$ in $\odot$.

Proof. It has been shown above that $\Gamma$ can be made continuous on any sufficiently small neighborhood in $\mathscr{O}^{\prime}$ by a suitable choice of the branch of the logarithms. This is also true for $\mathscr{Q}$. I shall prove this by showing that, if $\mu^{(0)}$ belongs to $\mathscr{O} \backslash \mathscr{C}^{\prime \prime}$, then $\Gamma_{1}+\Gamma_{2}$ has a finite limit as $\mu$ tends to $\mu^{(0)}$ in $\mathcal{C}^{\prime}$. Suppose that, for $\mu=\mu^{(0)}$, one has $P_{j}=P_{0 k}$ for $j$ varying in a subset $J$ of indices. It is obvious, from (4.8)(4.9), that, in that instance, the integrand density in the expression (4.4) of $\Gamma_{1}$ is no longer absolutely integrable. For such a reason, it is not possible to straightforward interchange the order of integration and limit as $\mu \rightarrow \mu^{(0)}$ in the right-hand side of (4.4). To cope with that problem, choose $\varepsilon_{j}$ small but finite, such that $P_{j}$ is contained in $B\left(P_{0 k_{j}}, \varepsilon_{j}\right)$ for any $j$ in $J$ and $B\left(P_{0 k_{j}}, \varepsilon_{j}\right)$ and $B\left(P_{0 k_{j^{\prime}}}, \varepsilon_{j^{\prime}}\right)$ have empty intersection for $j \neq j^{\prime}$ in $J$. In (4.4), the integral over $\Sigma$ is the sum of an integral over $\Sigma \backslash \cup_{\jmath \in J} B\left(P_{0 k_{\jmath}}, \varepsilon_{\jmath}\right)$ and one over $\cup_{j \in J} B\left(P_{0 k_{\jmath}}, \varepsilon_{\jmath}\right)$. In the first integral, one can interchange integration and limit as $\mu \rightarrow \mu^{(0)}$, since the integration domain is independent from $\mu$ and the singularities of the integrand density are well outside the integration domain. In the second integral, one can use the singular expansion of the integrand near the $P_{\jmath}$ 's and the $P_{0 k}$ 's. The resulting expression combines with $\Gamma_{2}$ in such a way that the limit as $\mu \rightarrow \mu^{(0)}$ exists finite. Let us illustrate this analysis in greater detail. The absolutely non-integrable part of the integrand density in (4.4) is given by

$$
\frac{\mu \partial Z_{j} \partial Z_{0 k_{j}}}{Z_{j} Z_{0 k_{j}}}=\bar{\partial}\left(\frac{\ln Z_{\jmath} \partial Z_{0 k_{\jmath}}}{Z_{0 k_{\jmath}}}\right),
$$

up to a factor $\nu_{j} \nu_{0 k_{j}} / 24 \pi$, where the identity $\bar{\partial} Z_{0 k_{j}}=0$ has been used (cf. Eq. (2.5) 
with $\mu=0$ ). By using (4.46), Stokes' theorem and (4.7), one finds

$$
\begin{aligned}
\int_{B\left(P_{0 k_{\jmath}}, \varepsilon_{\jmath}\right)} & \frac{d \bar{z} \wedge d z}{2 i} \frac{\mu \partial Z_{j} \partial Z_{0 k_{j}}}{Z_{j} Z_{0 k_{j}}} \\
= & \frac{1}{2 i} \int_{\partial B\left(P_{0 k_{j}}, \varepsilon_{j}\right) \backslash\left\{P^{*}\right\}} \frac{d z \partial Z_{0 k_{j}} \ln Z_{j}}{Z_{0 k_{\jmath}}}+\pi\left[\ln Z_{0 k_{j}}\left(P_{j}\right)-\ln Z_{0 k_{\jmath}}\left(P^{*}\right)\right] \\
& -\lim _{\varrho_{j} \rightarrow 0+} \frac{1}{2 i} \oint_{\partial B\left(P_{0 k_{\jmath}}, \varrho_{\jmath}\right)} \frac{d z \partial Z_{0 k_{j}} \ln Z_{j}}{Z_{0 k_{j}}} \\
= & \frac{1}{2 i} \int_{\partial B\left(P_{0 k_{j}}, \varepsilon_{j}\right) \backslash\left\{P^{*}\right\}} \frac{d z \partial Z_{0 k_{j}}}{Z_{0 k_{j}}}\left(\ln Z_{j}-\ln Z_{0 k_{\jmath}}\right)+i \pi^{2} \\
& +\pi\left[\ln Z_{0 k_{j}}\left(P_{j}\right)-\ln Z_{j}\left(P_{0 k_{\jmath}}\right)\right]
\end{aligned}
$$

In applying Stokes' theorem, one has to take into account the fact that $\ln Z_{j}$ has a branch cut emanating from $P_{\jmath}$ and intersecting $\partial B\left(P_{0 k_{\jmath}}, \varepsilon_{\jmath}\right)$ at some point $P^{*}$. The second term in the middle member of (4.47) is the contribution of the branch cut of $\ln Z_{j}$. The branch of $\ln Z_{0 k_{j}}$ is chosen so that its branch cut intersects $\partial B\left(P_{0 k_{j}}, \varepsilon_{j}\right)$ also at $P^{*}$. From (4.46)-(4.47), it follows that

$$
\begin{aligned}
\Lambda_{j}\left(\varepsilon_{\jmath}\right)= & \lim _{\mu \rightarrow \mu^{(0)}}\left\{\frac { 1 } { 2 4 \pi } \int _ { B ( P _ { 0 k _ { \jmath } , \varepsilon _ { \jmath } ) } } \frac { d \overline { z } \wedge d z } { 2 i } \left[2 \mathscr{B}_{0} \mu+2 \partial^{2} \mu+\partial \ln \omega_{0} \partial \mu+\partial \ln \omega \partial \mu\right.\right. \\
& \left.\left.+\partial \ln \omega_{0} \partial \ln \omega \mu\right]-\frac{\nu_{j} \nu_{0 k_{j}}}{24}\left[\ln Z_{0 k_{\jmath}}\left(P_{j}\right)-\ln Z_{j}\left(P_{0 k_{j}}\right)\right]\right\}
\end{aligned}
$$

exists finite in $\mathbf{C}$ with

$$
\Lambda_{j}\left(\varepsilon_{j}\right)=\frac{\nu_{j} \nu_{0 k_{j}}}{24}\left\{\frac{1}{2 \pi i} \oint_{\partial B\left(P_{0 k_{j}}, \varepsilon_{\jmath}\right)} \frac{d z \partial Z_{0 k_{\jmath}}}{Z_{0 k_{j}}}\left(\ln Z_{\jmath}{ }^{(0)}-\ln Z_{0 k_{j}}\right)+\pi i\right\}+O\left(\varepsilon_{\jmath}\right)
$$

where $Z_{j}{ }^{(0)}=Z_{j}\left(\mu^{(0)}\right)$. The contribution of order $\varepsilon_{j}$ comes from the non-singular part of the integrand density in the left-hand side. From (4.5) and the expansions (4.8)-(4.9), it follows that

$$
\Lambda_{0}\left(\varepsilon_{j}\right)=\Lambda_{0}=\lim _{\mu \rightarrow \mu^{(0)}}\left\{\Gamma_{2}+\frac{1}{24} \sum_{j \in J} \nu_{j} \nu_{0 k_{j}}\left[\ln Z_{0 k_{j}}\left(P_{j}\right)-\ln Z_{j}\left(P_{0 k_{j}}\right)\right]\right\}
$$


exists finite in C. Finally, from (4.48) and (4.49), one obtains that

$$
\begin{aligned}
\lim _{\mu \rightarrow \mu^{(0)}}\left(\Gamma_{1}+\Gamma_{2}\right)= & \lim _{\varepsilon_{\jmath} \rightarrow 0+}\left\{\frac{1}{24 \pi} \int_{\Sigma \backslash \cup_{\jmath} \in J B\left(P_{0 k_{\jmath}}, \varepsilon_{\jmath}\right)} \frac{d \bar{z} \wedge d z}{2 i}\right. \\
& \times\left[2 \mathscr{B}_{0} \mu+2 \partial^{2} \mu+\partial \ln \omega_{0} \partial \mu+\partial \ln \omega \partial \mu+\partial \ln \omega_{0} \partial \ln \omega \mu\right]^{(0)} \\
& \left.+\sum_{j \in J \cup\{0\}} \Lambda_{j}\left(\varepsilon_{j}\right)\right\}
\end{aligned}
$$

exists finite in $\mathbf{C}$. The existence and finiteness of the limit as the $\varepsilon_{j}$ 's tend to 0 of the surface integral follow from standard analytic arguments from the theory of Cauchy principal value and, crucially, from the fact that $\left|\mu^{(0)}\right|<1$ everywhere in $\Sigma$. The existence and finiteness of the limit as $\varepsilon_{j}$ tends to 0 of $\Lambda_{j}\left(\varepsilon_{j}\right)$ follow from $(4.48 \mathrm{~b})$ by inspection. From (4.50), it follows that the branches of the logarithms contained in the expression (4.5) of $\Gamma_{2}$ can be chosen so that the sum $\Gamma_{1}+\Gamma_{2}$ is continuous locally in 9 .

Next let us show that $\Gamma_{1}+\Gamma_{2}$ is singlevalued. The map $\mu \rightarrow P_{j}(\mu)$ is certainly continuous. As $\odot$ is simply connected by assumption, any loop in $\odot$ is contractible and so is the image of that loop by the map $P_{j}(\cdot)$. So, if $\mu$ is carried around a closed loop in $\odot, P_{j}(\mu)$ never winds around a non-trivial loop of $\Sigma$. Correspondingly, $\widehat{P}_{j}(\mu)$ describes a closed loop in $\Sigma_{\pi}$. For such a reason, the only multivaluedness of $\Gamma_{1}+\Gamma_{2}$ can arise when $\mu$ is carried along a closed loop in $\mathscr{Q}$ such that one or more of the $P_{\jmath}(\mu)$ 's wind around one or more of the $P_{0 k}$ 's. If such operation is performed, then the $\widehat{P}_{j}(\mu)$ 's with the same labels $j$ as the $P_{j}(\mu)$ 's wind the same number of times with the same orientation around certain points $\gamma_{k}\left(\widehat{P}_{0 k}\right)$ with the same labels $k$ as the $P_{0 k}$ 's, where the $\gamma_{k}$ 's are elements of the universal covering group Deck $\pi$ of $\pi$ (cf. Sect. 2). Then, one has

$$
\begin{gathered}
\nu_{j} \ln \left(\Omega / \omega_{0}\right)\left(\widehat{P}_{j}\right) \rightarrow \nu_{j} \ln \left(\Omega / \omega_{0}\right)\left(\widehat{P}_{j}\right)-2 \pi i \nu_{\jmath} \sum_{k} n_{\imath j} \nu_{0 k}, \\
\nu_{0 k} \ln \left(\omega / \Omega_{0}\right)\left(\widehat{P}_{0 k}\right) \rightarrow \nu_{0 k} \ln \left(\omega / \Omega_{0}\right)\left(\widehat{P}_{0 k}\right)+2 \pi i \nu_{0 k} \sum_{j} n_{i j} \nu_{\jmath},
\end{gathered}
$$

where the $n_{i j}$ 's are certain integers. From (4.51) and (4.5), it follows that $\Gamma_{1}+\Gamma_{2}$ is left unchanged, i.e. it is singlevalued.

The continuity of $\Gamma_{1}+\Gamma_{2}$ follows from its singlevaluedness and the fact that $\Gamma_{1}+\Gamma_{2}$ can be rendered continuous on any sufficiently small neighborhood in by a suitable choice of the branch of the logarithms. QED

v) $\Gamma(\mu)$ satisfies (4.1) in 9 .

Proof. The statement follows trivially from (4.45). Although (4.45) has been obtained for $\mu$ in $\mathscr{Q}^{\prime}$, it obviously extends by continuity to $\mathscr{Q}$. QED

vi) $\Gamma(\mu)$ satisfies (4.2) in (9.

Proof. Equation (4.42) is the key identity. Although (4.42) has been obtained for $\mu$ in $\mathscr{Q}^{\prime}$, it clearly extends by continuity to $\mathscr{Q}$ since all reference to $\omega_{0}$ has disappeared in the right-hand side. To compute $s \Gamma$, one replaces the operator $\delta$ by the Slavnov operator $s$ in (4.42) and uses the relations (3.5), (3.7), and (3.9) with $j=1$ together 
with the relations (4.36), (4.40). From (3.7), one finds readily

$$
s Z_{\jmath}=C \partial Z_{j} .
$$

Consistency of (3.9) and (4.52) requires that

$$
\begin{gathered}
s f_{j}(\zeta)=0, \\
s F_{j}(\zeta)=0 .
\end{gathered}
$$

Further, on has

$$
\begin{aligned}
(\bar{\partial}- & \mu \partial+(\partial \mu)) C\left[\mathscr{B}_{0}-\partial^{2} \ln \omega+\frac{1}{2}(\partial \ln \omega)^{2}\right] \\
= & \bar{\partial}\left[C\left(\mathscr{R}_{0}-\partial^{2} \ln \omega+\frac{1}{2}(\partial \ln \omega)^{2}\right)\right]-\partial\left[\mu C\left(\mathscr{B}_{0}-\partial^{2} \ln \omega+\frac{1}{2}(\partial \ln \omega)^{2}\right)\right] \\
& +C(\bar{\partial}-\mu \partial-2(\partial \mu))\left[-\mathscr{B}_{0}+\partial^{2} \ln \omega-\frac{1}{2}(\partial \ln \omega)^{2}\right]
\end{aligned}
$$

From (4.10) and (4.14), one has also

$$
(\bar{\partial}-\mu \partial-2(\partial \mu))\left[-\mathscr{R}_{0}+\partial^{2} \ln \omega-\frac{1}{2}(\partial \ln \omega)^{2}\right]=\left(\partial^{3}+2 \mathscr{B}_{0} \partial+\left(\partial \mathscr{B}_{0}\right)\right) \mu \text {. }
$$

The next step consists in substituting the identities (4.55) and (4.56) into (4.42) and eliminating the total derivative terms by means of Stokes' theorem. This yields a set of line integrals which are computed by a procedure similar to that used earlier. From (4.36), (2.5) and (4.7), one has

$$
\begin{aligned}
& \lim _{\varepsilon_{\jmath} \rightarrow 0+} \frac{1}{2 \pi i} \oint_{\partial B\left(P_{\jmath}, \varepsilon_{\jmath}\right)}(d z+\mu d \bar{z}) C\left(\mathscr{B}_{0}-\partial^{2} \ln \omega+\frac{1}{2}(\partial \ln \omega)^{2}\right) \\
& =\lim _{\varepsilon_{\jmath} \rightarrow 0+} \frac{1}{2 \pi i} \oint_{\partial B\left(P_{\jmath}, \varepsilon_{j}\right)}\left[\frac{d Z_{j}}{Z_{j}^{2}} \frac{\nu_{j}\left(\nu_{j}+2\right)}{2} C \partial Z_{j}+\frac{d Z_{j}}{Z_{j}} \nu_{j} \frac{\partial_{\zeta} f_{j}(0)}{f_{j}(0)} C \partial Z_{j}\right] \\
& =\frac{\nu_{\jmath}\left(\nu_{\jmath}+2\right)}{2} \frac{\partial\left(C \partial Z_{j}\right)}{\partial Z_{j}}\left(P_{j}\right)+\nu_{\jmath} \frac{\partial_{\zeta} f_{j}(0)}{f_{j}(0)} C \partial Z_{j}\left(P_{j}\right) .
\end{aligned}
$$

From (4.42), (4.52)-(4.54), (4.6c), (4.55)-(4.56), Stokes' theorem and (4.57), one verifies readily that $s \Gamma$ is given by (4.2). QED

Having shown the basic properties of $\Gamma(\mu)$, let us move to the computation of the derivative $\delta \Gamma(\mu) / \delta \mu$ which is an object of considerable salience. Physically, it represents the energy momentum tensor of the conformal field theory. Mathematically, it admits an interpretation as a holomorphic section of a certain holomorphic fiber bundle over Teichmueller space, as will be shown in the next section. $\delta \Gamma(\mu) / \delta \mu$ appears in the differential form of the Ward identity (4.2),

$$
(\bar{\partial}-\mu \partial-2(\partial \mu)) \frac{\delta \Gamma(\mu)}{\delta \mu}=\frac{-1}{12 \pi}\left(\partial^{3}+2 \mathscr{R}_{0} \partial+\left(\partial \mathscr{R}_{0}\right)\right) \mu,
$$

which follows readily from (4.2) and (3.5). This relation entails that

$$
\frac{\delta \Gamma(\mu)}{\delta \mu}=\frac{1}{12 \pi}\left[\mathscr{B}_{0}-\mathscr{B}_{\Gamma}(\mu)\right]
$$

where $\mathscr{B}_{\Gamma}(\mu)$ is an element of $\operatorname{Proj}(\Sigma, \mu)$ depending holomorphically on $\mu$ [8], defining a holomorphic section of $\operatorname{Schw}(\Sigma)$ (cf. Sect. 2). By using the expressions (4.6) of $\Gamma(\mu)$, it is possible to compute $\mathscr{B}_{\Gamma}(\mu)$ explicitly. From (4.58), it is evident 
that $\mathscr{R}_{\Gamma}(\mu)$ is determined up to the addition of an arbitrary holomorphic $\operatorname{Diff}_{c}(\Sigma)$ invariant section of $\mathscr{H}^{2}(\Sigma)$ on $\mathscr{O}$. This indetermination reflects the non-uniqueness of the functional $\Phi(\mu)$ appearing in (4.6).

Before proceeding to the calculation of $\mathscr{R}_{\Gamma}(\mu)$, it is necessary to state some rather general assumptions on the nature of the distributions that give the variations $\delta \omega(\mu)$ and $\delta \Omega(\mu)$ in terms of $\delta \mu$. The treatment of both variations is essentially identical. Let $\phi$ be either $\omega$ or $\Omega$. The assumptions are the following. i) The distributional kernel $\delta \phi(\mu)(P) / \delta \mu\left(P^{\prime}\right)$ is an ordinary function outside the diagonal $\left.\left\{P, P^{\prime}\right):\left(P, P^{\prime}\right) \in \Sigma_{\pi} \times \Sigma, \pi(P)=P^{\prime}\right\}$. ii) For fixed $P$ in $\Sigma_{\pi}, \delta \phi(\mu)(P) / \delta \mu\left(P^{\prime}\right)$ belongs to $H^{2}(\Sigma \backslash\{\pi(P)\}, \mu)$. iii) For fixed $P^{\prime}$ in $\Sigma, \delta \phi(\mu)(P) / \delta \mu\left(P^{\prime}\right)$ belongs to $H_{\pi}^{1}\left(\Sigma \backslash\left\{P^{\prime}\right\}, \mu\right)$. For $P^{\prime}$ approaching $\pi(P)$, one has the singular expansion

$$
\begin{aligned}
\frac{\delta \phi(\mu)(P)}{\delta \mu\left(P^{\prime}\right)}= & \frac{-1}{\pi} \partial Z(P) \partial Z\left(P^{\prime}\right)^{2} \\
& \times\left\{\frac{{ }^{\theta} \phi(\mu)(P)}{\left(Z\left(P^{\prime}\right)-Z(P)\right)^{2}}+\frac{\partial_{Z}{ }^{\theta} \phi(\mu)(P)}{Z\left(P^{\prime}\right)-Z(P)}+\text { regular terms }\right\} .
\end{aligned}
$$

Here, $Z$ is a coordinate of $\mathbf{A}(\mu)$ defined near $\pi(P)$ and $P^{\prime}$. (Recall that $Z(\pi(P))=$ $Z(P)$, where in the right-hand side $Z$ is viewed as a coordinate of $\mathbf{A}(\mu)$ in $\Sigma_{\pi}$ ). Further, ${ }^{\theta} \phi(\mu)=\phi(\mu) / \partial Z$. iv) $\delta \phi(\mu)$ is given by

$$
\delta \phi(\mu)(P)=\lim _{\varepsilon \rightarrow 0+} \int_{\Sigma \backslash B(\pi(P), \varepsilon)} \frac{d \bar{z} \wedge d z}{2 i} \frac{\delta \phi(\mu)(P)}{\delta \mu} \delta \mu .
$$

I could not prove that the above hypothesis on $\delta \phi(P) / \delta \mu\left(P^{\prime}\right)$ are fulfilled by an arbitrary $\phi$ but I have verified them in examples. Further, it should be noted that $\delta \phi(P) / \delta \mu\left(P^{\prime}\right)$ should have the same formal properties as the correlator of an energymomentum tensor operator and a $U(1)$ current density operator. So i-iii are expected to hold on physical grounds, (4.60) representing the standard operator product expansion. It is also possible to show that the properties i-iii follow from iv. However, since this is not really pertinent with the topic of the paper, I shall omit the details of the demonstration.

Now, one possesses all elements needed to write down the expression of the projective connection $\mathscr{R}_{\Gamma}(\mu)$ appearing in (4.59). For $\mu$ in $\mathscr{G}$, one has

$$
\mathscr{B}_{\Gamma}(\mu)=\partial^{2} \ln \omega(\mu)-\frac{1}{2}(\partial \ln \omega(\mu))^{2}-Q(\mu)-\frac{\delta \Phi(\mu)}{\delta \mu},
$$

where

$$
\begin{aligned}
Q(\mu)(P)= & \sum_{j} \mathscr{F}(\mu)\left(P, \widehat{P}_{j}(\mu) ; \nu_{j}(\mu)\right) \\
\mathscr{F}(\mu)\left(P, P^{\prime} ; \nu\right)= & \frac{\nu \pi}{2}\left\{\frac{1}{\Omega(\mu)\left(P^{\prime}\right)} \frac{\delta \Omega(\mu)\left(P^{\prime}\right)}{\delta \mu(P)}+\left(\frac{1}{\mathscr{\mathscr { D }}_{\Omega}{ }^{\nu} \omega(\mu)\left(P^{\prime}\right)} \mathscr{V}_{\Omega \nu P^{\prime}}\right.\right. \\
& \left.\left.-\frac{\nu-1}{\nu+1} \frac{\mathscr{D}_{\Omega}^{\nu+1} \omega(\mu)}{\left(\mathscr{D}_{\Omega}{ }^{\nu} \omega(\mu)\right)^{2}}\left(P^{\prime}\right) \mathscr{D}_{\Omega}{ }^{\nu-1}{ }_{P^{\prime}}\right) \frac{\delta \omega(\mu)\left(P^{\prime}\right)}{\delta \mu(P)}\right\} .
\end{aligned}
$$

Here, $\mathscr{D}_{\Omega}$ is the covariant derivative associated the polydromic $\mu$-holomorphic affine connection $\partial \ln \Omega(\mu) . \mathscr{R}_{\Gamma}(\mu)$ is everywhere smooth in spite of the fact that the first three contributions in the right-hand side of (4.62a) are singular at the zeros $P_{j}(\mu)$ of $\omega(\mu)$. 
Proof. The proof is based on the general variational formula (4.42). The strategy consists in expressing the integrated contributions in the right-hand side of (4.42) in terms of the variations $\delta \omega$ and $\delta \Omega$ and then in expressing those variations in terms of $\delta \mu$ by means of the integral formula (4.61) with $\phi=\omega, \Omega$. From (4.36), it is not difficult to obtain the following identities

$$
\begin{aligned}
\partial^{\nu_{j} \omega}= & \nu_{\jmath} !\left(\partial Z_{\jmath}\right)^{\nu_{j}+1}\left[f_{j}\left(Z_{\jmath}\right)+O\left(Z_{\jmath}\right)\right] \\
\partial^{\nu_{\jmath}+1} \omega= & \left(\nu_{\jmath}+1\right) !\left(\partial Z_{j}\right)^{\nu_{j}+2}\left[\left(1+\frac{\nu_{j}}{2}\right) \frac{\partial^{2} Z_{\jmath}}{\left(\partial Z_{j}\right)^{2}} f_{j}\left(Z_{j}\right)\right. \\
& \left.+\partial_{\zeta} f_{j}\left(Z_{\jmath}\right)+O\left(Z_{j}\right)\right], \\
\partial^{\nu_{\jmath}-1} \delta \omega= & \nu_{j} !\left(\partial Z_{j}\right)^{\nu_{\jmath}}\left[f_{j}\left(Z_{j}\right) \delta Z_{j}+O\left(Z_{j}\right)\right], \\
\partial^{\nu_{\jmath} \delta \omega=} & \left(\nu_{j}+1\right) !\left(\partial Z_{j}\right)^{\nu_{j}+1}\left[f_{j}\left(Z_{\jmath}\right)\left(\frac{\partial \delta Z_{j}}{\partial Z_{j}}+\frac{\nu_{\jmath}}{2} \frac{\partial^{2} Z_{j} \delta Z_{\jmath}}{\left(\partial Z_{\jmath}\right)^{2}}\right)\right. \\
& \left.+\partial_{\zeta} f_{j}\left(Z_{j}\right) \delta Z_{j}+\frac{1}{\nu_{j}+1} \delta f_{j}\left(Z_{j}\right)+O\left(Z_{j}\right)\right],
\end{aligned}
$$

From (4.36), (4.40) and (4.63)-(4.64), one finds that

$$
\begin{aligned}
\nu_{j}[ & \left.\left(\nu_{j}+2\right) \frac{\partial \delta Z_{\jmath}}{\partial Z_{j}}\left(P_{j}\right)+\frac{2 \partial_{\zeta} f_{\jmath}(0)}{f_{\jmath}(0)} \delta Z_{\jmath}\left(P_{j}\right)+\frac{\delta f_{j}(0)}{f_{j}(0)}+\frac{\delta F_{j}(0)}{F_{j}(0)}\right] \\
= & \nu_{\jmath}\left\{\frac{\delta \Omega}{\Omega}\left(\widehat{P}_{\jmath}\right)+\frac{1}{\partial^{\nu} \omega}\left[\partial^{\nu_{j}}-\frac{\nu_{j}\left(\nu_{j}+1\right)}{2} \partial \ln \Omega \partial^{\nu_{j}-1}\right.\right. \\
& \left.\left.-\frac{\nu_{\jmath}-1}{\nu_{j}+1} \frac{1}{\partial^{\nu_{\jmath}} \omega}\left(\partial^{\nu_{\jmath}+1} \omega-\frac{\left(\nu_{\jmath}+1\right)\left(\nu_{j}+2\right)}{2} \partial \ln \Omega \partial^{\nu_{j} \omega}\right) \partial^{\nu_{\jmath}-1}\right] \delta \omega\left(\widehat{P}_{\jmath}\right)\right\} \\
= & \nu_{j}\left\{\left(\frac{1}{\mathscr{D}_{\Omega} \nu_{\jmath} \omega} \mathscr{D}_{\Omega}{ }^{\nu_{\jmath}}-\frac{\nu_{j}-1}{\nu_{j}+1} \frac{\mathscr{D}_{\Omega} \nu_{j}+1 \omega}{\left(\mathscr{D}_{\Omega} \nu_{j} \omega\right)^{2}} \mathscr{D}_{\Omega}{ }^{\nu_{\jmath}-1}\right) \delta \omega\left(\widehat{P}_{\jmath}\right)+\frac{\delta \Omega}{\Omega}\left(\widehat{P}_{\jmath}\right)\right\} .
\end{aligned}
$$

In the very last step, I have exploited the fact $\partial^{\lambda} \omega\left(P_{j}\right)=0$ for $\lambda \leq \nu_{j}$ and $\partial^{\lambda} \delta \omega\left(P_{j}\right)=0$ for $\lambda \leq \nu_{j}-1$, as noticed earlier.

Next, one has to substitute the integral formula (4.61) with $\phi=\omega, \Omega$ into the right-hand side of (4.65) and express everything as a cut-off integral of a density. The latter step is not trivial, since it involves the exchange of integration and differentiation when $\partial^{\lambda}$ acts on the right-hand side of (4.61). Let $\nu \leq \nu_{j}$. Choose $\varrho>0$ small but finite. Then,

$$
\begin{aligned}
\left\{\partial^{\nu}{ }_{P} \lim _{\varepsilon \rightarrow 0+} \int_{\Sigma \backslash B(P, \varepsilon)} \frac{d \bar{z} \wedge d z}{2 i} \frac{\delta \omega(P)}{\delta \mu} \delta \mu\right\}_{P=P_{\mathcal{J}}} \\
=\int_{\Sigma \backslash B\left(P_{j}, \varrho\right)} \frac{d \bar{z} \wedge d z}{2 i}\left\{\partial_{P}^{\nu} \frac{\delta \omega(P)}{\delta \mu}\right\}_{P=P_{\mathcal{J}}} \delta \mu \\
\quad-\left\{\partial_{P}^{\nu} \lim _{\varepsilon \rightarrow 0+} \int_{B\left(P_{\jmath}, \varrho\right) \backslash B(P, \varepsilon)} \frac{d \bar{z} \wedge d z}{2 i} \frac{\delta \omega(P)}{\delta \mu} \delta \mu\right\}_{P=P_{\jmath}} .
\end{aligned}
$$


Now, I shall show that the second term in the right-hand side vanishes in the limit $\varrho \rightarrow 0+$. By using the expansion (4.60), the variational relation $(\bar{\partial}-\mu \partial-(\partial \mu)) \delta \phi=$ $\partial(\delta \mu \phi)$ with $\phi=\omega$ and (2.5), one finds that

$$
\begin{aligned}
\frac{\delta \omega(P)}{\delta \mu} & =\frac{-1}{\pi}\left\{\frac{\partial Z_{j}(P) \partial Z_{\jmath} \omega \delta \mu}{\left(Z_{j}(P)-Z_{j}\right)^{2}}+\text { regular terms }\right\} \\
& =\frac{-1}{\pi} \partial Z_{j}(P)\left\{\partial\left(\frac{\omega \delta \mu}{Z_{\jmath}(P)-Z_{j}}\right)-(\bar{\partial}-\mu \partial-(\partial \mu))\left(\frac{\delta \omega}{Z_{\jmath}(P)-Z_{\jmath}}\right)\right\}
\end{aligned}
$$

near $P_{j}$. One needs further the expansion

$$
\begin{aligned}
\partial^{\nu+1} & \left.\ln \left(Z_{j}(P)-Z_{\jmath}\right)\right|_{P=P_{\jmath}} \\
& =-\nu !\left(\partial Z_{j}\left(P_{\jmath}\right)\right)^{\nu+1}\left\{\frac{1}{Z_{j}{ }^{\nu+1}}+\frac{\nu+1}{2} \frac{\partial^{2} Z_{j}}{\left(\partial Z_{j}\right)^{2}}\left(P_{j}\right) \frac{1}{Z_{j}{ }^{\nu}}+O\left(\frac{1}{Z_{\jmath}{ }^{\nu-1}}\right)\right\} .
\end{aligned}
$$

From (4.36), the limit to be computed vanishes manifestly for all values of $\nu$ except for $\nu_{1}-1$ and $\nu_{j}$ because of the suppressing factor $Z_{\jmath}^{\nu_{3}}$ and $Z_{\jmath}^{\nu_{3}-1}$ appearing in $\omega$ and $\delta \omega$, respectively. By using (4.67), Stokes' theorem, (2.8), (4.68), (4.7), and (4.27), one obtains, for $\nu=\nu_{\jmath}, \nu_{\jmath}-1$,

$$
\begin{aligned}
& \lim _{\varrho \rightarrow 0+}\left\{\partial_{P}^{\nu} \lim _{\varepsilon \rightarrow 0+} \int_{B\left(P_{\jmath}, \varrho\right) \backslash B(P, \varepsilon)} \frac{d \bar{z} \wedge d z}{2 i} \frac{\delta \omega(P)}{\delta \mu} \delta \mu\right\}_{P=P_{J}} \\
& =\left.\lim _{\varrho \rightarrow 0+} \frac{1}{2 \pi i} \oint_{\partial B\left(P_{\jmath}, \varrho\right)} \frac{d Z_{\jmath} \delta \omega}{\partial Z_{\jmath}} \partial_{P}^{\nu} \ln \left(Z_{\jmath}(P)-Z_{\jmath}\right)\right|_{P=P_{\jmath}}+\partial^{\nu} \delta \omega\left(P_{\jmath}\right) \\
& +\left.\lim _{\varrho \rightarrow 0+} \frac{1}{2 \pi i} \oint_{\partial B\left(P_{\jmath}, \varrho\right)} d \bar{z} \omega \delta \mu \partial_{P}^{\nu} \ln \left(Z_{\jmath}(P)-Z_{\jmath}\right)\right|_{P=P_{j}} \\
& =-\nu !\left(\partial Z_{j}\left(P_{j}\right)\right)^{\nu+1} \lim _{\varrho \rightarrow 0+} \frac{1}{2 \pi i} \oint_{\partial B\left(P_{\jmath}, \varrho\right)}\left\{\frac{d Z_{j}}{Z_{\jmath}{ }^{\nu-\nu_{\jmath}+2}} \nu_{\jmath} f_{j}\left(Z_{j}\right) \delta Z_{j}\right. \\
& +\frac{d Z_{j}}{Z_{\jmath}{ }^{\nu-\nu_{j}+1}}\left[f_{j}\left(Z_{j}\right)\left(\frac{\partial \delta Z_{\jmath}}{\partial Z_{j}}+\frac{\nu_{\jmath}(\nu+1)}{2} \frac{\partial^{2} Z_{\jmath}}{\left(\partial Z_{j}\right)^{2}}\left(P_{j}\right) \delta Z_{j}\right)\right. \\
& \left.\left.+\partial_{\zeta} f_{j}\left(Z_{j}\right) \delta Z_{j}+\delta f_{j}\left(Z_{j}\right)\right]\right\}+\partial^{\nu} \delta \omega\left(P_{\jmath}\right) \\
& -\nu !\left(\partial Z_{j}\left(P_{j}\right)\right)^{\nu+1} \lim _{\varrho \rightarrow 0+} \frac{1}{2 \pi i} \oint_{\partial B\left(P_{j}, \varrho\right)} \frac{d \bar{z} \delta \mu \partial Z_{j}}{Z_{j}^{\nu-\nu_{j}+1}} f_{j}\left(Z_{j}\right) \\
& =0 \text {. }
\end{aligned}
$$

In the very last step, I have used (4.64). It follows immediately from here that one can interchange the order of differentiation at $P_{\jmath}$ and cut-off integration. From this remark, (4.61) with $\phi=\omega, \Omega$ and (4.65), one obtains (4.62) immediately. The smoothness of $\mathscr{R}_{\Gamma}$ can be verified straightforwardly by plugging (4.36), (4.40) and the expansion (4.60) with $\phi=\omega, \Omega$ into (4.62) and then by checking that all the singular terms cancel out. QED 
To conclude this section, I shall provide am explicit construction of the polydromic 1-differential $\Omega . \Omega(\mu)$ may be expressed in terms of Fay's $\sigma$ field [29] as follows:

$$
\Omega(\mu)(P)=\left(\frac{\sigma(\mu)(P)}{\sigma(\mu)\left(\widehat{P}^{(1)}(\mu)\right)}\right)^{2 / g} \omega^{(2)}(\mu)\left(\widehat{P}^{(1)}(\mu)\right),
$$

where $\omega^{(1)}$ and $\omega^{(2)}$ are distinct $\operatorname{Diff}_{c}(\Sigma)$-invariant sections of $\mathscr{H}^{1}(\Sigma)$ and $\widehat{P}^{(1)}(\mu)$ is a lift of a zero $P^{(1)}(\mu)$ of $\omega^{(1)}(\mu)$ to ${ }^{\prime} \Sigma_{\pi}$ depending continuously on $\mu$.

\section{Discussion and Conclusion}

It is interesting to study the Polyakov action in terms of Teichmueller space and of holomorphic fiber bundles thereof. Recall that Teichmueller space Teich $(\Sigma)$ is the quotient $\operatorname{Beltr}(\Sigma) / \operatorname{Diff}_{c}(\Sigma)$ and is a complex manifold of complex dimension $3 g-3$. $\operatorname{Beltr}(\Sigma)$ may thus be viewed as a $\operatorname{Diff}_{c}(\Sigma)$ principal bundle on $\operatorname{Teich}(\Sigma)$. This bundle is smoothly but not holomorphically trivial $[8,27]$. The following can be shown.

A Polyakov action $\Gamma$ can be defined globally on $\operatorname{Beltr}(\Sigma)$, the functional dependence on the fibers of the latter being determined by the Ward identity (4.1). This property is evident also from the fact that the diffeomorphism invariant effective action and the local counterterm that subtract the Weyl anomaly are globally defined in $\operatorname{Beltr}(\Sigma)$.

Proof. Let $\Gamma_{\alpha}(\mu)$ be a Polyakov action defined on a $\operatorname{Diff}_{c}(\Sigma)$-invariant open simply connected subset $\odot_{\alpha}$ of $\operatorname{Beltr}(\Sigma)$. Set

$$
\xi_{\alpha \beta}(\mu)=\exp \left(\Gamma_{\alpha}(\mu)-\Gamma_{\beta}(\mu)\right), \quad \text { on } \mathscr{Q}_{\alpha} \cap \mathscr{Q}_{\beta} \neq \emptyset \text {. }
$$

From (4.1)-(4.2), it follows that

$$
\begin{gathered}
\frac{\delta \xi_{\alpha \beta}(\mu)}{\delta \bar{\mu}}=0, \\
s \xi_{\alpha \beta}(\mu)=0 .
\end{gathered}
$$

It is clear from (5.1)-(5.3) that $\xi_{\alpha \beta}(\mu)$ defines a holomorphic line bundle on Teich $(\Sigma)$, which is necessarily holomorphically trivial, since $\operatorname{Teich}(\Sigma)$ is a contractible domain of holomorphy [27]. Hence, for each patch $\alpha$, there is a functional $\eta_{\alpha}(\mu)$ such that

$$
\begin{aligned}
\frac{\delta \eta_{\alpha}(\mu)}{\delta \bar{\mu}} & =0, \\
s \eta_{\alpha}(\mu) & =0, \\
\xi_{\alpha \beta}(\mu) & =\eta_{\alpha}(\mu) / \eta_{\beta}(\mu) .
\end{aligned}
$$

Now, redefine $\Gamma_{\alpha}(\mu)$ as

$$
\Gamma_{\alpha}(\mu) \rightarrow \Gamma_{\alpha}(\mu)-\ln \eta_{\alpha}(\mu) .
$$

This operation fixes the undetermined functional $\Phi_{\alpha}(\mu)$ which appears in the general expression (4.6). Thus, $\Gamma_{a}(\mu)$ is the restriction to $Q_{\alpha}$ of a well-defined functional $\Gamma(\mu)$ defined for every $\mu$ in $\operatorname{Beltr}(\Sigma)$ and satisfying (4.1)-(4.2), that is $\Gamma(\mu)$ is a Polyakov action on $\operatorname{Beltr}(\Sigma)$. QED

Hubbard space $\operatorname{Hubb}(\Sigma)$ is the quotient $\operatorname{Schw}(\Sigma) / \operatorname{Diff}_{c}(\Sigma)$ (cf. Sects. 2 and 3) and is a holomorphically trivial holomorphic fiber bundle over Teich $(\Sigma)$ [27]. Every holomorphic Diff $c(\Sigma)$-invariant section of $\operatorname{Schw}(\Sigma)$ defines a holomorphic section of $\operatorname{Hubb}(\Sigma)$. The following holds. 
The section $\mathscr{R}_{\Gamma}(\mu)$ of $\operatorname{Schw}(\Sigma)$ associated to $\Gamma(\mu)$ via (4.59) is holomorphic and $\operatorname{Diff}_{c}(\Sigma)$-invariant, hence it defines a holomorphic section of $\operatorname{Hubb}(\Sigma)$.

Proof. Holomorphy is obvious from (4.1) and (4.59). The proof of $\operatorname{Diff}_{c}(\Sigma)$ invariance requires some argument. Let us denote by $\delta$ the differentiation operator on the manifold $\operatorname{Beltr}(\Sigma) . \delta$ anticommutes with $s, \delta s+s \delta=0$. From (4.2), one has

$$
\begin{aligned}
\delta s \Gamma(\mu)= & \frac{1}{12 \pi}\left\{\int_{\Sigma} \frac{d \bar{z} \wedge d z}{2 i} \delta C(\mu)\left(\partial^{3}+2 \mathscr{B}_{0} \partial+\left(\partial \mathscr{R}_{0}\right)\right) \mu\right. \\
& \left.-\int_{\Sigma} \frac{d \bar{z} \wedge d z}{2 i} \delta \mu\left(\partial^{3}+2 \mathscr{R}_{0} \partial+\left(\partial \mathscr{R}_{0}\right)\right) C(\mu)\right\} .
\end{aligned}
$$

By using the identity

$$
\delta s \mu=-\delta \mu \partial C(\mu)+\partial \delta \mu C(\mu)+(\bar{\partial}-\mu \partial+(\partial \mu)) \delta C(\mu),
$$

which follows readily from (3.5), one obtains

$$
\begin{aligned}
s \delta \Gamma(\mu)= & s \int_{\Sigma} \frac{d \bar{z} \wedge d z}{2 i} \delta \mu \frac{\delta \Gamma(\mu)}{\delta \mu} \\
= & \frac{1}{12 \pi}\left\{\int _ { \Sigma } \frac { d \overline { z } \wedge d z } { 2 i } \delta \mu \left[\left(2\left(\mathscr{R}_{0}-\mathscr{R}_{\Gamma}(\mu)\right) \partial+\left(\partial\left(\mathscr{R}_{0}-\mathscr{R}_{\Gamma}(\mu)\right)\right)\right) C(\mu)\right.\right. \\
& \left.\left.+s \mathscr{R}_{\Gamma}(\mu)\right]-\int_{\Sigma} \frac{d \bar{z} \wedge d z}{2 i} \delta C(\mu)\left(\partial^{3}+2 \mathscr{R}_{0} \partial+\left(\partial \mathscr{R}_{0}\right)\right) \mu\right\}
\end{aligned}
$$

Now, from (5.8) and (5.10), one finds

$$
\int_{\Sigma} \frac{d \bar{z} \wedge d z}{2 i} \delta \mu\left[s \mathscr{R}_{\Gamma}(\mu)-\left(\partial^{3}+2 \mathscr{R}_{\Gamma}(\mu) \partial+\left(\partial \mathscr{R}_{\Gamma}(\mu)\right)\right) C(\mu)\right]=0 .
$$

Hence, $\mathscr{R}_{\Gamma}$ satisfies (3.8), which is an equivalent statement of the $\operatorname{Diff}_{c}(\Sigma)$-invariance of $\mathscr{R}_{\Gamma}$. QED

The origin and nature of the $S L(2, \mathrm{C})$ symmetry.

For any Beltrami differential $\mu$ in $\operatorname{Beltr}(\Sigma)$, one may consider the projective structure $\mathbf{P}\left(\mu, \mathscr{B}_{\Gamma}(\mu)\right)$ associated to the element $\left(\mu, \mathscr{R}_{\Gamma}(\mu)\right)$ of $\operatorname{Schw}(\Sigma)$ (cf. Sect. $2)$. In terms of the coordinates $Z_{\Gamma}(\mu)$ of $\mathbf{P}\left(\mu, \mathscr{R}_{\Gamma}(\mu)\right)$, the relation

$$
\mathscr{R}_{\Gamma}(\mu)=\left\{Z_{\Gamma}(\mu), z\right\}
$$

holds (cf. Eq. (2.14)). $\mathscr{R}_{\Gamma}(\mu)$ is invariant under variations of $Z_{\Gamma}(\mu)$ of the form

$$
\delta Z_{\Gamma}(\mu)=\alpha+\beta Z_{\Gamma}(\mu)+\gamma Z_{\Gamma}(\mu)^{2},
$$

where $\alpha, \beta, \gamma$ are arbitrary local a-antimeromorphic functions. This is the passive form of the SL(2,C) symmetry in conformal field theory on higher genus Riemann surfaces. The infinitesimal generators of all transformations of this type are given by

$$
\begin{aligned}
& J_{n}^{-}(\mu)=\bar{z}^{-n} \otimes \frac{1}{\partial Z_{\Gamma}(\mu)} \partial, \\
& J_{n}^{0}(\mu)=\bar{z}^{-n} \otimes-2 Z_{\Gamma}(\mu) \frac{1}{\partial Z_{\Gamma}(\mu)} \partial, \\
& J_{n}^{+} \cdot(\mu)=\bar{z}^{-n} \otimes Z_{\Gamma}(\mu)^{2} \frac{1}{\partial Z_{\Gamma}(\mu)} \partial .
\end{aligned}
$$


and form a SL(2,C) loop algebra on the field $\mathbf{C}$ of complex numbers attached to each point $\mu$ of the manifold $\operatorname{Beltr}(\Sigma)$.

The $\operatorname{SL}(2, \mathbf{C})$ symmetry has also an active form. To any projective structure $\mathbf{P}(\mu, \mathscr{B})$ (cf. Sect. 2) there is associated a flat $\operatorname{PSL}(2, \mathbf{C})$ vector bundle $\sigma(\mu, \mathscr{R})$ in canonical fashion $[28,30]$. An endomorphism $\tau$ of $\sigma(\mu, \mathscr{B})$ is a triple of fields $\tau^{+}, \tau^{0}$ and $\tau^{-}$ forming a section of the adjoint representation $\operatorname{adj} \sigma(\mu, \mathscr{R})$ of $\sigma(\mu, \mathscr{B})$. It is possible to check that if $\tau$ is an endomorphism then

$$
\xi_{\tau}=\left(\tau^{+}-2 \tau^{0} Z+\tau^{-} Z^{2}\right) / \partial Z
$$

is a well defined element of $C^{-1,0}(\Sigma)$. To any such $\tau$ there is associated an infinitesimal action on the coordinates of $\mathbf{P}(\mu, \mathscr{R})$ given by

$$
\delta_{\tau} Z=\xi_{\tau} \partial Z
$$

Through (2.7) and (2.14), this action can be extended to the Beltrami differential $\mu$ and to the projective connection $\mathscr{B}$ by

$$
\begin{aligned}
\delta_{\tau} \mu & =(\bar{\partial}-\mu \partial+(\partial \mu)) \xi_{\tau}, \\
\delta_{\tau} \mathscr{R} & =\left(\partial^{3}+2 \mathscr{R} \partial+(\partial \mathscr{R})\right) \xi_{\tau} .
\end{aligned}
$$

The action of an endomorphism $\tau$ of $\sigma(\mu, \mathscr{B})$ equals the diffeomorphism action of the following element of Lie $\operatorname{Diff}_{c}(\Sigma)$

$$
\eta_{\tau}=\frac{\xi_{\tau}-\mu \bar{\xi}_{\tau}}{1-\mu \bar{\mu}}
$$

since $\xi_{\tau}=\eta_{\tau}+\mu \bar{\eta}_{\tau}$. Using the identity $\partial^{3}+2 . \mathscr{R} \partial+(\partial \mathscr{B})=(\partial Z)^{2}\left(\frac{1}{\partial Z} \partial\right)^{3}$

following from (2.14), it is immediate to see that, if $\tau$ is a-antiholomorphic, then $\delta_{\tau} \mathscr{R}=0$. The active form of the $\operatorname{SL}(2, \mathbf{C})$ is given by a-antiholomorphic endomorphisms of the flat PSL(2,C) vector bundle $\sigma_{\Gamma}(\mu)=\sigma\left(\mu, \mathscr{R}_{\Gamma}(\mu)\right)$. From the above discussion and the $\operatorname{Diff}_{c}(\Sigma)$-invariance of $\mathscr{R}_{\Gamma}(\mu)$ as a section of $\operatorname{Schw}(\Sigma)$, it follows that

$$
\left.\delta \mathscr{R}_{\Gamma}(\mu)\right|_{\delta \mu=\delta_{\tau} \mu}=0 .
$$

One concludes that: i) the active form of the SL(2,C) symmetry consists of the a-antiholomorphic gauge transformations of the flat $\operatorname{PSL}(2, \mathbf{C})$ vector bundle $\sigma_{\Gamma}(\mu)$ and is equivalent to a class of infinitesimal diffeomorphisms and ii) the invariance of energy-momentum tensor $T_{\Gamma}(\mu)$ under such symmetry is a consequence of the vanishing of the diffeomorphism anomaly for infinitesimal diffeomorphisms of such type. It should be noticed that there is no proof that the Polyakov action itself is invariant under the $\operatorname{SL}(2, \mathrm{C})$ symmetry on a surface of high genus, unlike what happens for the planar topology.

Several problems remain open and call for further investigation. First, one should verify that the expression (4.6) of $\Gamma(\mu)$ resumes the perturbative series yielded by renormalized field theory. Doing this is certainly going to be arduous. In fact, this involves on one hand the expansion of $\Gamma(\mu)$, as given by (4.6), in a formal power series in the Beltrami differential $\mu$ and the correct identification of the distributional kernels $\left\langle T_{\Gamma}(1) \ldots T_{\Gamma}(n)\right\rangle$ involved at $n^{\text {th }}$ order in $\mu$ in the expansion and, on the other, the elaboration of Feynman's algorithm for any given model and the calculation of 
the renormalized $n$ point connected Green functions $\langle T(1) \ldots T(n)\rangle$ of the energymomentum tensor operator $T$. One expects that $\left\langle T_{\Gamma}(1) \ldots T_{\Gamma}(n)\right\rangle=\langle T(1) \ldots T(n)\rangle$ up to renormalization ambiguities.

For conformal field theories on the infinite plane, this endeavor has been initiated in $[23,24]$ (see also [8]). In that case, one can show that there is a unique renormalization satisfying the conformal Ward identity (4.58) and that, in fact, $\left\langle T_{\Gamma}(1) \ldots T_{\Gamma}(n)\right\rangle=$ $\langle T(1) \ldots T(n)\rangle$ exactly. This uniqueness of the renormalization does not hold for conformal models on a compact surface without boundary. The underlying reason for this fact is that on the plane the operator $\bar{\partial}-\mu \partial-2(\partial \mu)$ appearing in the conformal Ward identity has no zero modes while on a genus $g>1$ Riemann surface it has $3 g-3$ linearly independent zero modes. Such zero modes reflect indeed the renormalization ambiguities mentioned above.

Not all solutions of the Ward identity are physical. It is thus important to find constraints or conditions which select the admissible solutions. In this respect, modular covariance should play a prominent role. Regrettably, at the present stage of development of the subject, it is not clear how this could be carried out.

Acknowledgements. I wish to voice my gratitude to D. Buturovic, F. Gieres, M. Knecht, S. Lazzarini, F. Thuillier, G. Venturi and K. Yoshida for many helpful discussions and steady encouragement. In particular, I wish to stress my indebtedness to R. Stora, who introduced me to the topic of this paper and has been a constant source of suggestions and ideas. This paper is dedicated to the memory of Dino Zucchini.

\section{References}

1. Nelson, P.: Phys. Rep. 149, 337 (1987) and references therein

2. D’Hoker, E., Phong, D.H.: Rev. Mod. Phys. 60, 917 (1988) and references therein

3. Seiberg, N.: Lecture at the 1990 Yukawa International Seminar on Common Trends in Mathematics and Quantum Field Theories, Kyoto, Japan (1990) and at the Cargese meeting Random surfaces, quantum gravity and strings. Cargese, France (1990) and references therein

4 D'Hoker, E.: Preprint UCLA/91/TEP/35 (1991) and references therein

5. Polyakov, A.: Phys. Lett. B103, 207 (1981); Phys. Lett. B103, 211 (1981)

6. Friedan, D.: Les Houches lectures 1982, R. Stora and J.B. Zuber (eds.), Amsterdam: NorthHolland 1984

7. Eguchi, T., Ooguri, H.: Nucl. Phys. B282, 308 (1987)

8. Lazzarini, S.: Doctoral thesis, LAPP Annecy-le-Vieux (1990) and references therein

9. Gieres, F.: Gen. Relat. Gravit. 22, 889 (1990)

10. Delduc, F., Gieres, F.: Class. Quant. Grav. 7, 1907 (1990)

11. Knecht, M., Lazzarini, S., Thuillier, F.: Phys. Lett. B251, 279 (1990)

12. Lazzarini, S., Stora, R.: Talk at the $13^{\text {th }}$ John Hopkins Workshop on Current Problems in High Energy Particle Theory: Knots, Topology and Quantum Field Theories. Florence, Italy (1989). Proceedings, 549

13. Stora, R.: Talk at the 1990 Yukawa International Seminar on Common Trends in Mathematics and Quantum Field Theories, Kyoto, Japan (1990). In Prog. Theor. Phys. 102, 373 (1990)

14. Belavin, A.A., Knizhnik, V.G.: Phys. Lett. B168, 201 (1986)

15. Knecht, M., Lazzarini, S., Stora, R.: Phys. Lett. B262, 25 (1991); Phys. Lett. B273, 63 (1991)

16. Polyakov, A.: Mod. Phys. Lett. A2, 893 (1987)

17. Knizhnik, V.G., Polyakov, A., Zamolodchickov, A.B.: Mod. Phys. Lett. A3, 819 (1988)

18. Bershadsky, M., Ooguri, H.: Commun. Math. Phys. 126, 49 (1989)

19. Verlinde, E., Verlinde, H.: Lecture at Superstrings 89, Trieste, Italy (1989). Proceedings, 422

20. Verlinde, H.: Nucl. Phys. B337, 652 (1990)

21. Bonora, L., Martinelli, M., Viallet, C.M.: Phys. Lett. 197B, 335 (1987)

22. Baulieau, L., Bellon, M.: Phys. Lett. B196, 142 (1987) 
23. Becchi, C.: Nucl. Phys. B304, 513 (1988)

24. Becchi, C., Piguet, O.: Nucl. Phys. B315, 153 (1989)

25. Zucchini, R.: Phys. Lett. B260, 296 (1991)

26. Yoshida, K.: Mod. Phys. Lett. A4, 71 (1989); Int. J. Mod. Phys. A5, 4559 (1990)

27. Nag. S.: The complex analytic theory of Teichmueller spaces. Canadian Mathematical Society Series of Monographs and Advanced Tests, New York: Wiley 1988 and references therein

28. Gunning, R.: Lectures on Riemann surfaces. Princeton, NJ: Princeton University Press 1966 and references therein

29. Fay, J.D.: Theta Functions on Riemann surfaces, Lecture Notes in Mathematics, Vol. 352, BerlinHeidelberg-New York: Springer Verlag 1973

30. Gunning, R.: Lectures on Vector Bundles on Riemann surfaces. Princeton, NJ: Princeton University Press 1967 and references therein

Note added in proof. After this paper was completed, I became aware of ref. [31] where a similar calculation is presented for the supertorus.

[31] Ader, J.-P., Kachkachi, H.: Preprint LTPB 92-7, to appear in Class. Quant. Grav.

Communicated by K. Gawedzki 
